\title{
Loudspeaker Analysis: A Radar Based Approach
}

\author{
Alessio Izzo ${ }^{(}$, Ludovico Ausiello $^{(}{ }^{(}$Carmine Clemente $^{(}$, Senior Member, IEEE, \\ and John J. Soraghan, Senior Member, IEEE
}

\begin{abstract}
Recently, radar based micro-Doppler signature analysis has been successfully applied in various sectors including defence, biomedical, and automotive. This article presents the novel use of radar micro-Doppler for loudspeaker analysis. The approach offers the potential benefits of characterizing the mechanical motion of a loudspeaker in order to identify defects and design issues. Compared with acoustic based approaches, the use of radar allows reliable measurements in an acoustically noisy end of a production line. In addition, when compared with a laser vibrometric approach, the use of radar micro-Doppler reduces the number of measurements required and provides direct access to the information of the metallic components of the loudspeaker. In the paper experimental results and analysis of the micro-Doppler signatures of loudspeakers using low cost radar systems are presented. Based on Thiele\&Small parameters, the voice coil displacement is modelled and micro-Doppler signatures for a single tone and a sine sweep stimulus are presented. Furthermore, in order to characterize the speaker with a single radar measurement, a methodology to measure mechanical frequency response of loudspeakers is also shown.
\end{abstract}

Index Terms—Radar, micro-Doppler, micro-Doppler analysis, loudspeaker analysis, industrial processes.

\section{INTRODUCTION}

$\mathbf{L}$ OUDSPEAKERS condition monitoring is an important topic in audio manufacturing. Laser based analysis tools have been shown [1] to yield significantly better results compared to traditional acoustic ones. The former approach is more frequently used in advanced markets like automotive audio components and systems, while the latter is widely used in R\&D and manufacturing of acoustic transducers and consumer products (e.g. loudspeakers or audio products). However, both acoustic and laser analysis have technical and practical limitations that we show in this paper does not apply to the use of our radar based method. The effectiveness of acoustic End-Of-Line tests (EOL) or acoustic measurements is limited by the surrounding environment; as it normally

Manuscript received August 27, 2019; revised October 8, 2019; accepted October 9, 2019. This work was supported in part by the Engineering and Physical Sciences Research Council (EPSRC) under Grant EP/K014307/1 and in part by the MOD University Defence Research Collaboration in Signal Processing. The associate editor coordinating the review of this article and approving it for publication was Prof. Piotr J. Samczynski. (Corresponding author: Alessio Izzo.)

A. Izzo, C. Clemente, and J. J. Soraghan are with the Centre for Signal and Image Processing (CeSIP), Department of Electronic and Electrical Engineering, University of Strathclyde, Glasgow G1 1XW, U.K. (e-mail: alessio.izzo@strath.ac.uk; carmine.clemente@strath.ac.uk; j.soraghan@strath.ac.uk).

L. Ausiello is with the School of Media Arts and Technology, Solent University, Southampton SO14 0YN, U.K. (e-mail: Iudovico.ausiello@ solent. ac.uk).

Digital Object Identifier 10.1109/JSEN.2019.2946987 requires specifically designed insulated booths or silent areas for the signal-to-noise ratio of audio data to be meaningful. There are two main limitations when laser-based scanner vibrometer systems (Scanning Vibrometer System (SCN) [1]) are used in place of the traditional acoustic approach. The first is the requirement of a very large sets of measurements (up to almost 3000 points) to fully characterize a loudspeaker and its non linearities, thus being a serious time consuming activity. The second is the limitation due to the presence of any physical obstacle in the line of sight between the laser source and the membrane (or acoustic source) under test [2], [3].

Interest in micro-Doppler analysis has grown in the past decades, reaching a plethora of sectors and applications [4], [5]. Targets detection and classification has been improved thanks to the micro-Doppler signature, becoming an important radar based tool in defence application. A novel model-based automatic classification algorithm for helicopters was presented in [6], [7]. In [8], the micro-Doppler signature has been extracted from spectrogram and cepstrograms in order to discriminate birds and small unmanned aerial vehicles (UAVs). The micro-Doppler signature has been used even in more challenging scenario, as in [9] where the capability of micro-Doppler-based recognition in the specific challenge of distinguishing between warheads and confusing objects has been shown. Thanks to radar at more affordable prices, the micro-Doppler analysis became interesting even for civilian application. For example, in bio-medical field, 
the total human energy expenditure for walking and running activities is estimated by using the micro-Doppler signature, as shown in [10]. In automotive, micro-Doppler analysis has been used to classify pedestrian activity for Automatic Drive Assistant Systems (ADAS) [11]. The interest in micro-Doppler suggests that this technology is reliable, and that it is worth investigating it in further applications domains, such as the acoustic one. In [12], the authors introduce for the first time a novel approach based on radar micro-Doppler to analyse and measure the return from loudspeaker. This approach is motivated by the potential cost effectiveness and operational advantages that a radar based approach could introduce over acoustic and laser based ones. With respect to the traditional acoustic measurement, a radar based approach is not affected by the acoustic environmental factors, allowing its use for End of Line (EoL) test. Unlike the SCN system, the micro-Doppler has the ability to cope with visual occlusion due to plastic parts and the capability of separation metallic components of a loudspeaker from non metallic ones through the use of the back-scattering intensity.

In this paper a novel approach to measure loudspeaker characteristic is proposed exploiting low cost radar sensors and the micro-Doppler signatures. The novelty of this paper can be summarised as follow:

- A model for the radar return from a loudspeaker based on the Thiele and Small parameters.

- A methodology to measure mechanical frequency response of loudspeakers in order to characterise the speaker with a single radar measurement.

The remainder of the paper is organised as follows. The most common embodiment of electrodynamic transducer will be introduced in Section II, where an overview of electrodynamic theory and traditional acoustic measurement of a speaker will be given. In Section III, the concept of the micro-Doppler will be introduced. While in Section III-A the micro-Doppler signature of a speaker playing a single tone is analysed, in Section III-B the micro-Doppler signature of speaker playing a chirp signal is investigated. By using a chirp signal, the concept of mechanical frequency response will be introduced in order to characterize the speaker with a single measurement, in Section III-C. Results of experimental acquisitions will be compared with the simulated data in Section IV-A and IV-B, for both single tone and sine sweep analysis, in order to validate the expected micro-Doppler modulations. In Section IV-C the matched filter approach is applied to real data. Finally in Section V, conclusions and future developments are proposed.

\section{LOUDSPEAKER KINEMATIC}

A magnetic type transducer is a device able to convert an electrical signal into sounds. Belonging to this class of devices are the electro-dynamic or moving coil loudspeaker. Among different type of transducers [13], each one relying on different working principle, in this paper only direct radiator loudspeaker type is considered, where a cross sectional view is shown in Figure 1.

The cone or diaphragm is made from a suitably light and stiff material; most of its stiffness comes from its profile.

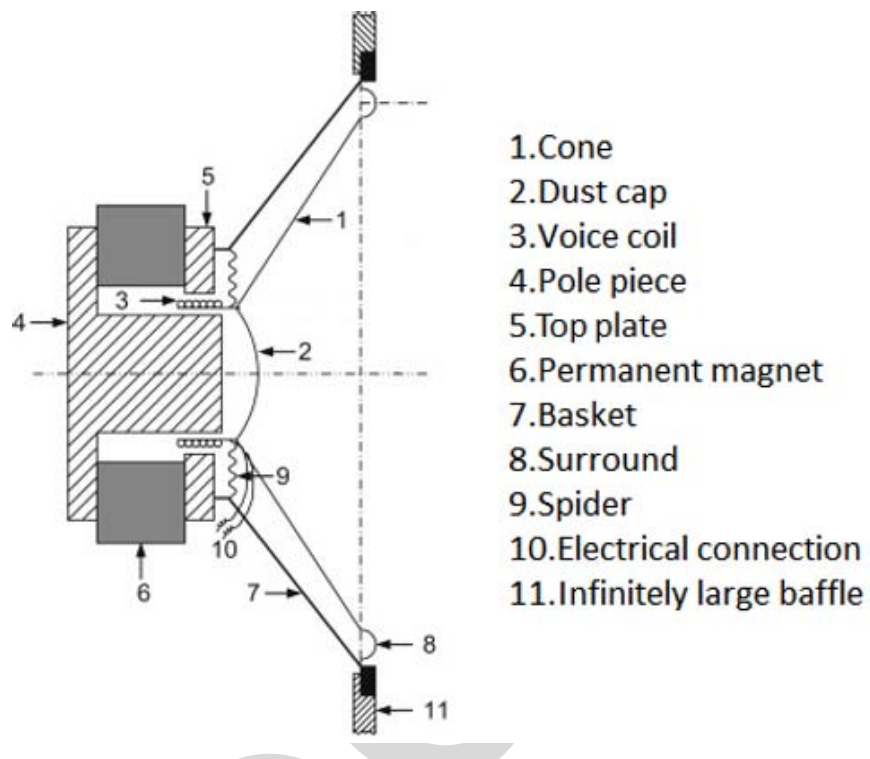

Fig. 1. Cross sectional sketch of a direct-radiator loudspeaker [13].

The profile can be designed as a straight line (a real cone) or curved. In order to prevent metallic dust falling into the magnetic gap a dust cap is placed in the centre; the dust cap is also useful to prevent sound from the back of the diaphragm to leak through the outer world. The coil is located in the gap of a magnetic path, comprising a pole piece and top plate, where the magnetic flux is produced by a permanent magnet, which is held in place by a basket structure. A surround and a spider are used to support the diaphragm at the rim and near the voice coil, respectively, so that it is free to move only in an axial direction. In general, sound from the back of the cone exits through holes in the basket, while sound from the back of the dust cap leaks through the magnetic gap and spider, which often presents holes or porosity, before exiting through the basket. When an audio signal is applied to the voice coil, the resulting current creates a magnetomotive force which interacts with the air-gap flux of the permanent magnet and causes a translatory movement of the voice coil and, hence, of the cone to which it is attached.

Sound waves are produced by the motion of the cone that displaces the air molecules at its surface. The loudness of the sound is, therefore, dependant on the acoustic pressure radiated by the membrane, proportional to the velocity, by which the cone moves and pushes the surrounding air [13]. The most widely used models for loudspeakers dynamics, assume that below $1 \mathrm{kHz}$ the drivers operate in what is referred to as the "piston mode," meaning that in the considered range of frequencies, the driver behaves as a rigid body. This assumption is not always verified, as measurements show that real drivers are never rigid. It is practically impossible to realise a perfect piston except for a small range of frequencies, which is related to the physical dimension of the diaphragm [2], [3], [14]. In this frequency band, force factor, stiffness and inductance introduce non linearities, generating spectral components that are not present in the input signal. An indication of the non linearities inherent in the system is given by the amplitude of the displacement. 


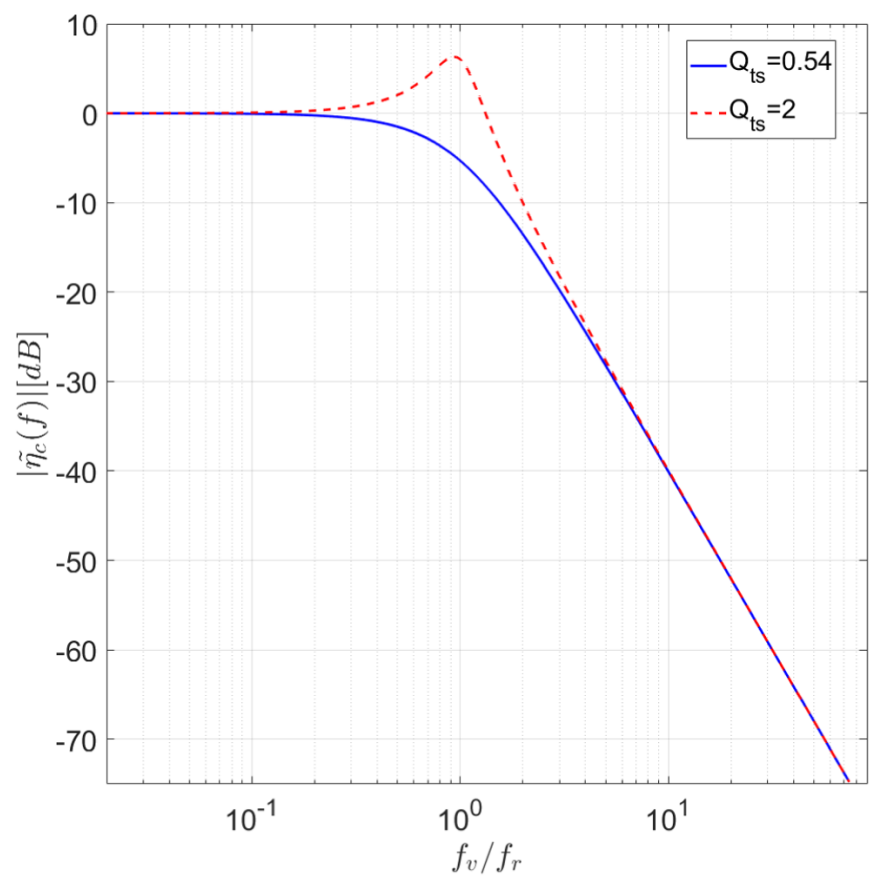

Fig. 2. Normalized voice coil displacement, with $Q_{t s}=0.54$ (blue line) and $Q_{t s}=2$ (red line).

Due to the dynamical analogies, the differential equations explaing the mechanical and acoustical behaviour can be solved by electrical circuit theory. Thus, with the assumption of rigid body motion, the displacement of a loudspeaker can be computed as function of the frequency of the stimulus by considering the electro-mechanical components responsible of the dynamic response of the transducer, known as Thiele and Small (T\&S) parameters [13]. In this way the voice coil displacement $\tilde{\eta}_{c}$, function of the acoustic frequency $f_{v}$, may be written as:

$$
\tilde{\eta}_{c}\left(f_{v}\right)=\frac{\tilde{e}_{g}}{2 \pi f_{r} B l Q_{e s}}\left|\gamma_{c}\left(f_{v}\right)\right|
$$

where $\tilde{e}_{g}$ is the voltage at the speaker's terminals, $B l$ is the force factor (magnetic flux density $B$ multiplied by the length of the wire $l), f_{r}$ is the resonance frequency of the speaker and $\gamma_{c}\left(f_{v}\right)$ is a dimensionless frequency response function given by:

$$
\gamma_{c}\left(f_{v}\right)=\frac{1}{1-\frac{f_{v}^{2}}{f_{r}^{2}}+j \frac{f_{v}}{f_{r} Q_{t s}}}
$$

where $Q_{t s}$ represents the total damping effect, composed by the electrical damping $Q_{e s}$ and the mechanical damping $Q_{m s}$, and $j$ is the imaginary unit. Equation (1) describes the frequency dependent behaviour of the loudspeaker displacement. The normalized voice coil displacement for different values of $Q_{t s}$ is shown in Figure 2. It can be noted how the total damping affects the displacement behaviour around $f_{v} / f_{r}=1$, while the displacement is virtually constant at $f_{v} / f_{r} \leq 1 / 3$ and proportional to $1 / f_{v}^{2}$ at $f_{v} / f_{r} \geq 3$.

Depending on the amplitude of the displacement, the transducer will generate distorted signals that can be classified as linear (low displacement amplitude) and non linear (high displacement amplitude) distortion. Both of them are regarded as regular distortions because they are accepted within the design process and are results of optimization process giving the best compromise with other constraints (weight, cost, size). On the other hand, irregular distortions are non acceptable defects in a loudspeaker passing the EOL tests. They are generated by defects caused during the manufacturing process, ageing and other external factor such as overload and temperature. A rubbing voice coil, buzzing parts, loose particles and air leaks are typical loudspeaker defects which produce irregular distortion, quite audible and not acceptable, generally defined as "rub \& buzz". In a woofer, for example, at higher frequencies (e.g. above $1 \mathrm{kHz}$ ), the cone itself is not rigid and should be modelled as a flexible system. The vibrations travel transversally along the cone surface in what is generally referred to as cone break up. This behaviour generally becomes a dominant factor when the wavelength of the sound in air is comparable to or less than twice cone diameter [2], [3]. Above this frequency, radial and rocking modes are natural vibration patterns of the membrane, producing non linear or undesired output. Furthermore, the presence of any irregularities (e.g. mass distribution) produced in the manufacturing/assembling process, and/or diaphragms subjected to asymmetric acoustic loads enhance this phenomena. This becomes even more critical in small drivers such as headphones or micro-speakers, where small irregularities in the stiffness, mass and magnetic field distributions can affect dramatically the dynamic behaviour of these tiny structures [3], [14], [15]. The resulting distortion depends highly on the amplitude and type of the stimulus. A well known technique commonly used in audio environment to completely characterise the system with a single, fast and easy measurement was introduced in [16]-[18]. It is based on exponentially swept sine signal defined as:

$$
x(t)=\sin \left[\frac{2 \pi f_{1} T}{\ln \left(\frac{f_{2}}{f_{1}}\right)}\left(\left(\frac{f_{2}}{f_{1}}\right)^{\frac{t}{T}}-1\right)\right]
$$

where $T$ is the length of the sine sweep in seconds, and $f_{1}$ and $f_{2}$ the starting and ending frequencies, respectively. This technique has the ability to separate the non-linear (distortion) responses from the linear response of the system. When the measured signal $y(t)$ is convolved with an inverse filter $g(t)$, namely the time reversal version of the test signal $x(t)$, the linear response compresses to an almost perfect impulse, with a delay equal to the length of the test signal. Simultaneously, the harmonic distortion responses compress to other smaller impulses, located at precise time delays occurring earlier than the impulse response. Applying a suitable time window it is possible to extract just the portion required, containing only the linear response or the distortion products. Thus, a Fourier transform can be applied, and both linear and non linear (harmonics) frequency responses can be displayed.

Having briefly introduced some of the aspects of the electrodynamic transducer motion, and how to acoustically characterize the behaviour of a speaker, further experimental analysis will be shown in the next sections focusing mainly on the rigid body motion of the loudspeaker at low frequencies in 


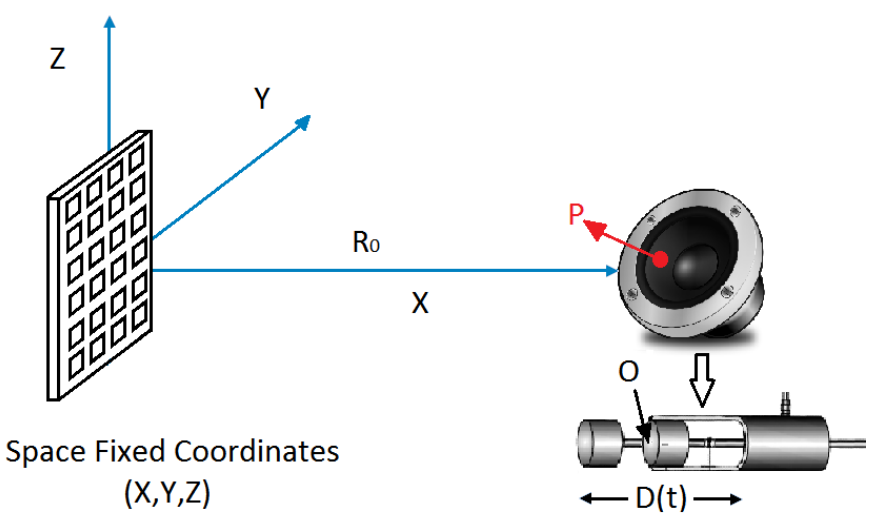

Fig. 3. Geometry for the radar and generic vibrating point: the motion of a speaker can be described as rigid body motion having a piston mode when the input to the loudspeaker is a signal with frequency range up to $1 \mathrm{kHz}$.

order to detect, confirm and characterize its behaviour through radar micro-Doppler signature.

\section{Micro-Doppler Analysis}

An operating loudspeaker present a complex scenario of moving parts which can generate a multifaceted pattern of vibrations. Radar sensor can be used to identify the vibration pattern of the transducer. To fully understand and interpret correctly the radar micro-Doppler phenomena in complex and realistic scenario, the basics of the micro-Doppler will be introduced in Section III-A where the single tone signal is used as stimulus, while in Section III-B the micro-Doppler signature of a speaker playing the exponential sine sweep in (3) is analysed. Finally, in Section III-C the radar based mechanical characterization of the speaker will be introduced. For all these analyses, a sampling frequency $f_{s}=22 \mathrm{kHz}$ is considered.

\section{A. Single Tone Analysis}

Radar micro-Doppler effect can be understood by considering target's micro-motions. In coherent radars, the range variations cause a phase change in the returned signal from a target. Thus, the Doppler frequency shift, rapresenting the change of phase function over time, can be used to detect vibrations or rotations of structures in a target. In Figure 3 the geometry used to analyse the micro-Doppler induced by a vibrating target is shown [4], [5].

The receiving Doppler from a target as a function of time is modelled as follows:

$$
s_{r}(t)=\rho \exp \left\{j\left[2 \pi f_{0} t+\Phi(t)\right]\right\}
$$

where $\rho$ is the reflectivity of the vibrating point scatterer, $f_{0}$ is the carrier frequency of the transmitted signal and $\Phi(t)$ is the time varying phase change of the vibrating scatterer. Letting $R_{0}$ be the distance between the radar and the speaker's initial position $O$, then the range function varies with time due to the speaker micro-motion:

$$
R(t)=R_{0}+D(t)
$$

Assuming an arbitrary point of the cone located in $P$ vibrates with sinusoidal frequency $f_{v}$ and maximum displacement $\tilde{\eta}_{c}\left(f_{v}\right)$, the displacement function will be of the kind:

$$
D(t)=\tilde{\eta}_{c}\left(f_{v}\right) \sin \left(2 \pi f_{v} t\right)
$$

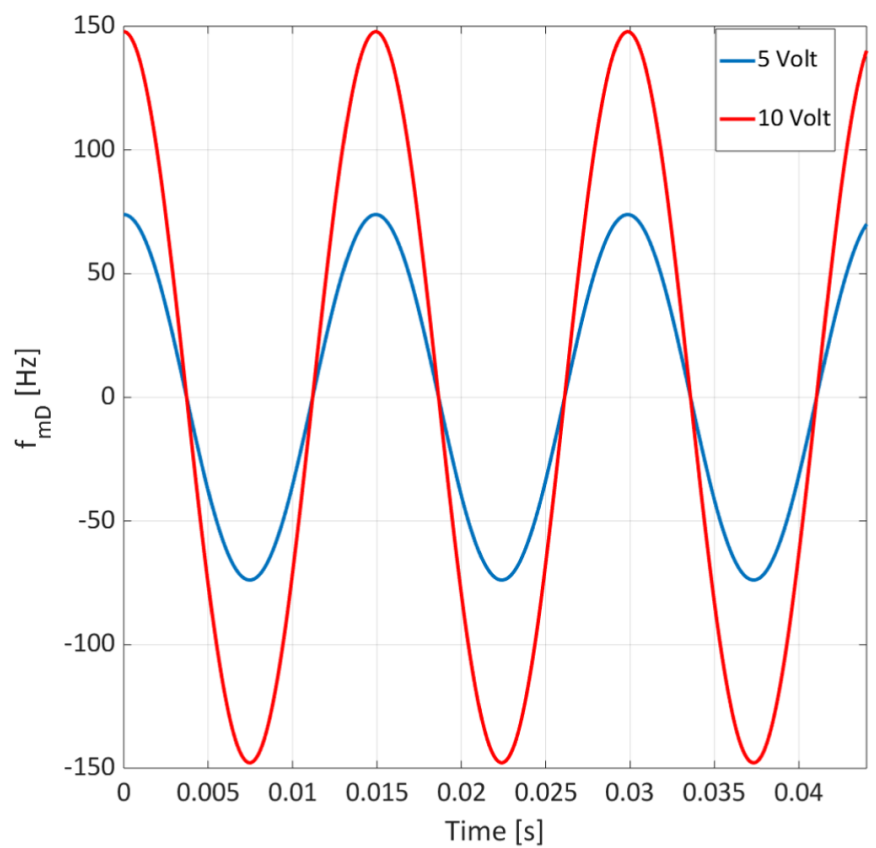

Fig. 4. Theoretical micro Doppler of a speaker moving at its resonance frequency of $f_{V}=f_{r}=67 \mathrm{~Hz}$ with output voltage of $5 \mathrm{~V}$ and $10 \mathrm{~V}$, modelled as a flat circular plate having maximum displacement of $\tilde{\eta}_{c, 5 \mathrm{~V}}=1.1 \mathrm{~mm}$ and $\tilde{n}_{c, 10 \mathrm{~V}}=2.2 \mathrm{~mm}$.

while assuming the radar being in the line of the sight with the speaker [4], [5]. Then, the time varying phase can be written as:

$$
\Phi(t)=\beta R(t)=\beta\left[R_{0}+\tilde{\eta}_{c}\left(f_{v}\right) \sin \left(2 \pi f_{v} t\right)\right]
$$

where

$$
\beta=\frac{4 \pi}{\lambda}
$$

with $\lambda$ the wavelength of the transmitted signal. Substituting (7) in (4) the received signal can be expressed as [12]:

$$
\begin{aligned}
s_{r}(t)=\rho \exp & \left\{j \frac{4 \pi R_{0}}{\lambda}\right\} \\
& \times \exp \left\{j 2 \pi f_{0} t+j \frac{4 \pi \tilde{\eta}_{c}\left(f_{v}\right)}{\lambda} \sin \left(\omega_{v} t\right)\right\}
\end{aligned}
$$

where $\omega_{v}=2 \pi f_{v}$. In order to simulate a received radar signal, the backscattering coefficient $\rho$ [4], [5] relative to the only vibrating metallic component, namely the voice coil, is modelled as flat circular plate and calculated as:

$$
\rho=\frac{4 \pi^{3} r^{4}}{\lambda^{2}}
$$

with $\lambda$ the radar signal wavelength and $r$ is the radius of voice coil. From (9), the derivative of the second phase term leads to the expression of the micro-Doppler shift:

$$
f_{m D}(t)=\frac{1}{2 \pi} \frac{d \Phi}{d t}=\frac{4 \pi}{\lambda} \tilde{\eta}_{c}\left(f_{v}\right) f_{v} \cos \left(2 \pi f_{v} t\right)
$$

In Figure 4 the theoretical micro Doppler of a speaker moving at its resonance frequency of $67 \mathrm{~Hz}$, with output voltage of $5 \mathrm{~V}$ and $10 \mathrm{~V}$, is shown.

From (1), the theoretical displacement $\tilde{\eta}_{c}$ at $5 \mathrm{~V}$ and $10 \mathrm{~V}$ of output voltage is computed. With a $\tilde{\eta}_{c, 5 \mathrm{~V}}=1.1 \mathrm{~mm}$ and $\tilde{\eta}_{c, 10 \mathrm{~V}}=2.2 \mathrm{~mm}$, the maximum Doppler shift achievable is $73.90 \mathrm{~Hz}$ and $147.80 \mathrm{~Hz}$, respectively. 


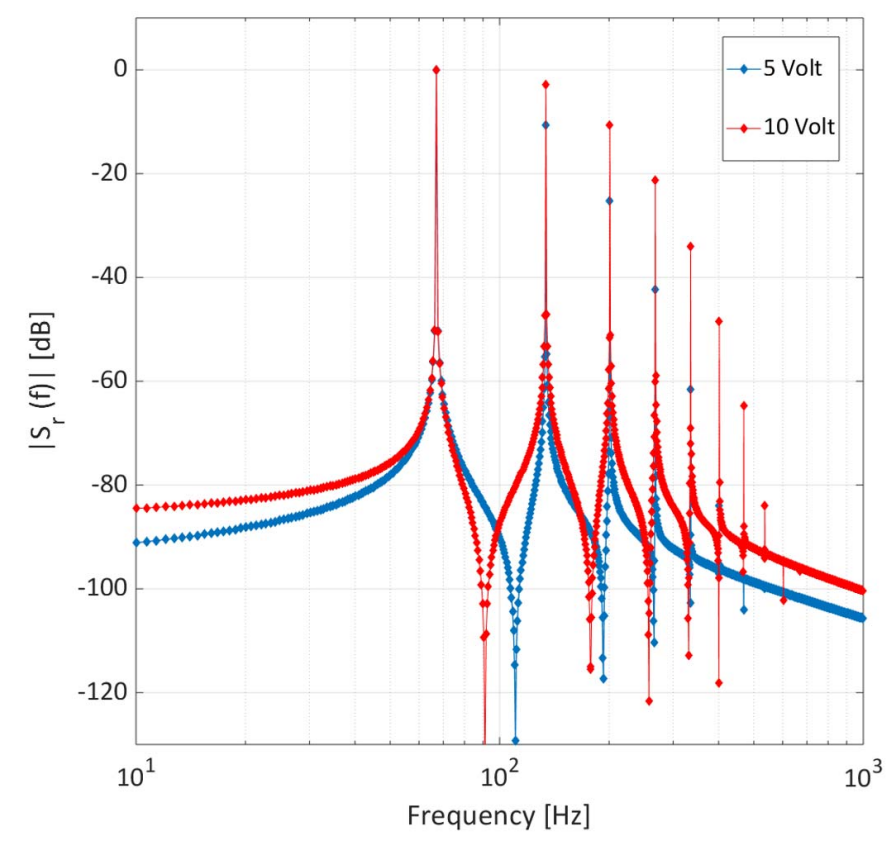

Fig. 5. Normalised spectrum of the simulated received signal of a speaker moving at its resonance frequency of $f_{V}=67 \mathrm{~Hz}$ with output voltage of $5 \mathrm{~V}$ and $10 \mathrm{~V}$, modelled as a flat circular plate having maximum displacement of $\tilde{\eta}_{c, 5 \mathrm{~V}}=1.1 \mathrm{~mm}$ and $\tilde{\eta}_{c, 10 \mathrm{~V}}=2.2 \mathrm{~mm}$.

The spectrum of a typical simulated received radar signal is shown in the Figure 5, for $5 \mathrm{~V}$ and $10 \mathrm{~V}$ of applied voltage. By Fourier analysis the vibration frequency of the coil can be detected, where the number of visible harmonics depends from the displacement amplitude, directly related to the micro Doppler. This result is in complete agreement with loudspeaker modelling theory. Loudspeakers and other kinds of actuators which produce sounds or vibrations behave differently at small and high displacement amplitudes. The dependency of the displacement amplitude is an indication of non linearities inherent in the system. As the displacement amplitude increases, particularly at low frequencies, the most dominant non linearities effects are introduced by stiffness $K_{m s}\left(\tilde{\eta}_{c}\right)$ (reciprocal of the compliance $C_{m s}\left(\tilde{\eta}_{c}\right)$ ), force factor $B l\left(\tilde{\eta}_{c}\right)$ and inductance $L_{e}\left(\tilde{\eta}_{c}\right)$, function of the displacement $\tilde{\eta}_{c}$. A second non linear effect is the generation of additional spectral components which are not in the exciting stimulus; these components are generally multiple of the fundamental frequency and thus labelled as harmonic and intermodulations distortion [3].

From this simple set of equations in (7),(9) and (11) describing the micro-Doppler signature we can deduct the capability to retrieve information about the behaviour, anomalies and failures of a loudspeaker from the radar returned.

While the spectral composition of a signal varies as function of the time, the conventional Fourier transform cannot provide a time dependent spectral description. Thus, a joint time-frequency distribution provides more insight into the time-varying behaviour of the signal. The squared magnitude of the Short-Time Fourier Transform [6], [8] of the received radar signal, namely the spectrogram $\chi(\nu, \kappa)$, is used to

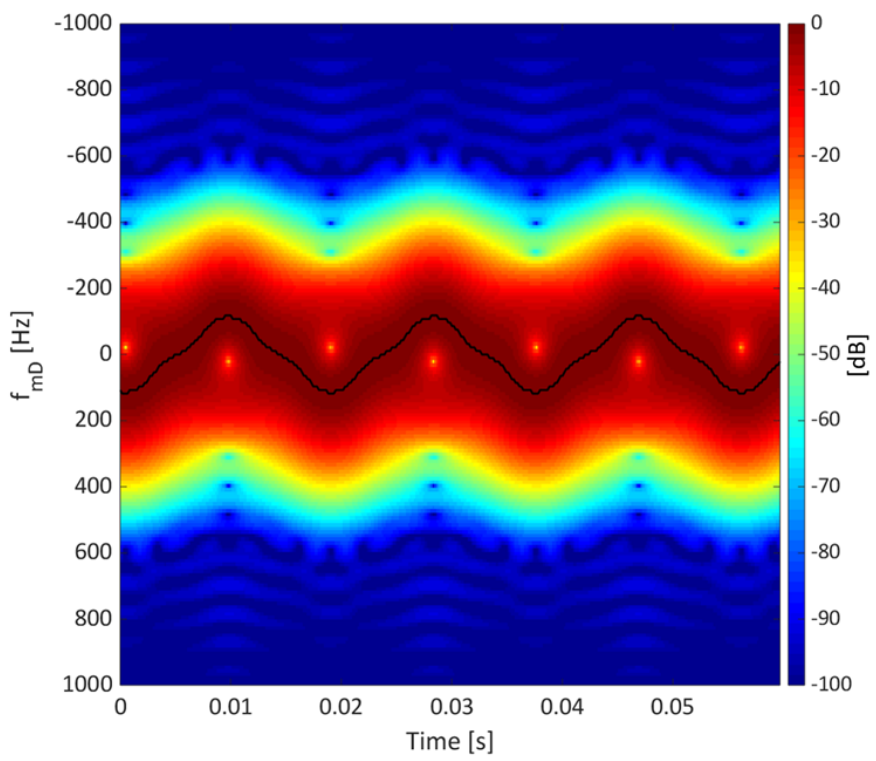

(a) Blackman-Harris window of $11.6 \mathrm{~ms}$

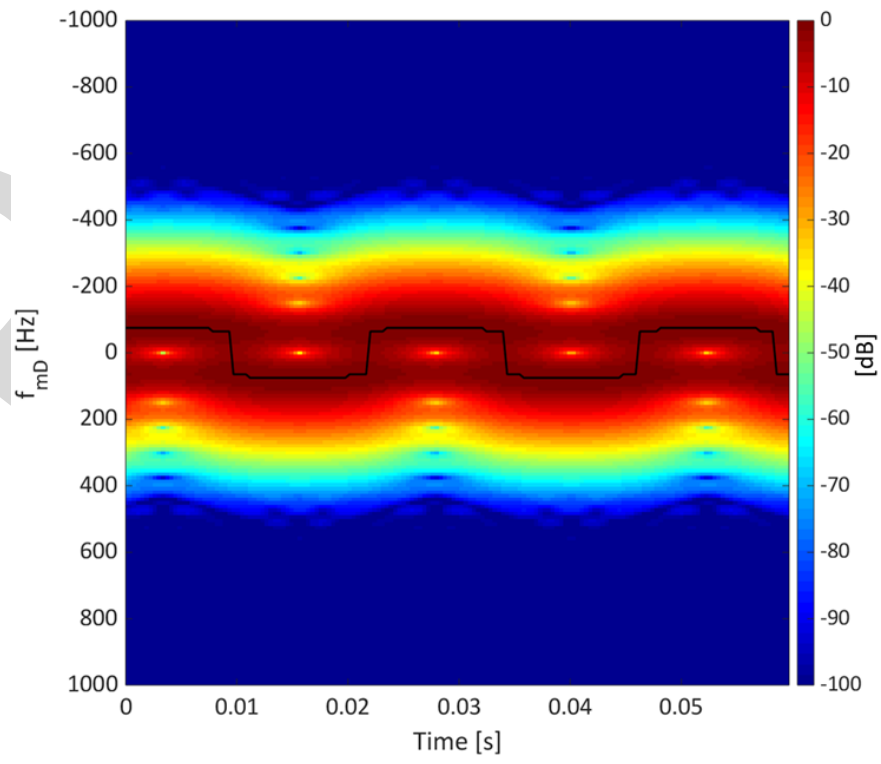

(b) Blackman-Harris window of $23.2 \mathrm{~ms}$

Fig. 6. Magnitude square of the spectrogram of the simulated received radar signal from a speaker moving at its resonance frequency $f_{V}=f_{r}=$ $67 \mathrm{~Hz}$ with output voltage of $5 \mathrm{~V}$, modelled as a flat circular plate having maximum displacement $\tilde{\eta}_{c, 5 \mathrm{~V}}=1.1 \mathrm{~mm}$. The maximum Doppler shift is highlighted with a black line.

examine the time-frequency distribution as:

$$
\begin{aligned}
\chi(v, \kappa) & =\left|\operatorname{STFT}\left\{s_{r}(n)\right\}\right|^{2} \\
& =\left|\sum_{n=0}^{N-1}\left(s_{r}(n) h(n-\kappa) \exp ^{\left(\frac{-j 2 \pi v n}{N}\right)}\right)\right|^{2}
\end{aligned}
$$

where $\kappa=0 \ldots, K-1, v$ is the normalised frequency and $h(n)$ is a discrete window function of choice [7], [11].

In Figure 6, spectrograms of the simulated received radar signal of a speaker moving at its resonance frequency $f_{v}=$ $f_{r}=67 \mathrm{~Hz}$ with output voltage of $5 \mathrm{~V}$ are shown. For a better 


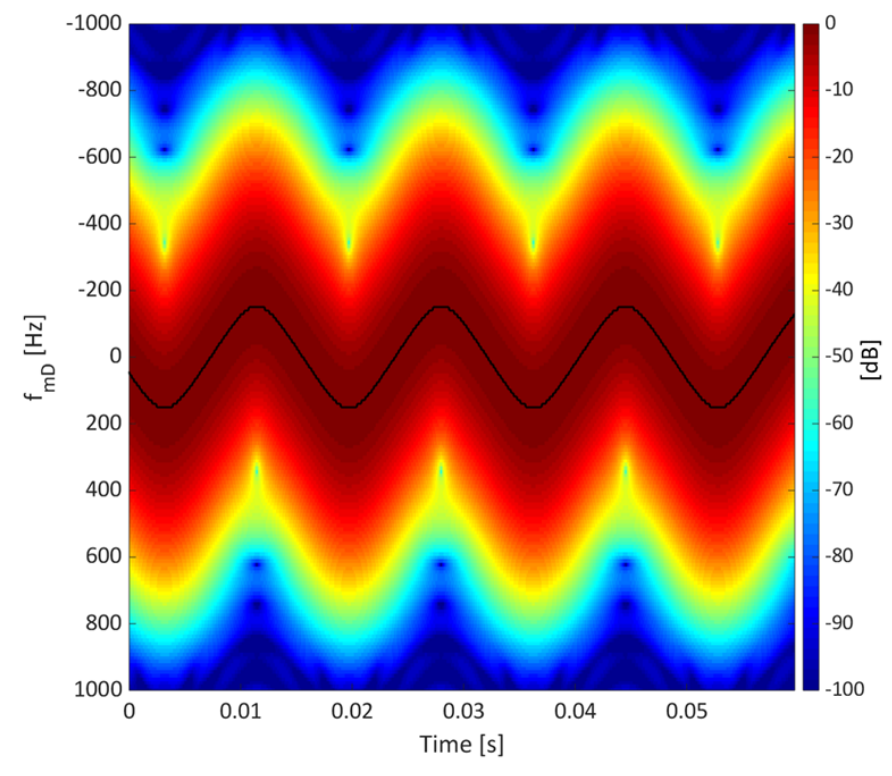

Fig. 7. Magnitude square of the spectrogram of the simulated received radar signal from a speaker moving at its resonance frequency $f_{v}=f_{r}=$ $67 \mathrm{~Hz}$ with output voltage of $10 \mathrm{~V}$, modelled as a flat circular plate having maximum displacement $\tilde{\eta}_{c, 10 \mathrm{~V}}=2.2 \mathrm{~mm}$, with Blackman-Harris window of $5.8 \mathrm{~ms}$. The maximum Doppler shift is highlighted with a black line.

understanding of the reader, the maximum Doppler shift is highlighted with a black line. In Figure 6(a), the spectrogram with a Blackman-Harris window of $11.6 \mathrm{~ms}$, confirming the sinusoidal-like motion. Due to the trade off between time-frequency resolution, the Doppler shift is bigger than the theoretical one. A better frequency resolution can be achieved by increasing the window length to $23.2 \mathrm{~ms}$, as shown in Figure 6(b) where a Doppler shift of $75 \mathrm{~Hz}$ is found, in agreement with the theoretical one. With an output voltage of $10 \mathrm{~V}$, the spectrogram in Figure 7 reveals a maximum Doppler shift of $150 \mathrm{~Hz}$, in agreement with what is expected from theory in (11).

\section{B. Sine Sweep Analysis}

Using the chirp signal $x(t)$ in (3), the ideal received radar signal $s_{r}(t)$ in baseband is modelled as:

$$
s_{r}(t)=\rho \exp \left\{j \frac{4 \pi \tilde{\eta}_{c}\left(f_{v}(t)\right)}{\lambda} x(t)\right\}
$$

with the displacement $\tilde{\eta}_{c}$ be a time varying function of $f_{v}(t)$, as described in (1). For an exponential sine sweep, the instantaneous vibration frequency $f_{v}(t)$ is defined as:

$$
f_{v}(t)=f_{1} k^{t}=f_{1}\left(\frac{f_{2}}{f_{1}}\right)^{\frac{t}{T}}
$$

with $k$ the exponential chirp rate. Depending on the behaviour of the displacement, the micro Doppler will show a different envelope, strictly related to voice coil motion. In case of constant displacement $\tilde{\eta}_{c}$ during the sweep, the maximum micro Doppler increases linearly with the frequency of the stimulus, with fixed modulation index $\Upsilon=\beta \tilde{\eta}_{c}$, as in (11). In Figure 8 the maximum micro Doppler for different fixed displacement at different vibration frequencies are shown.

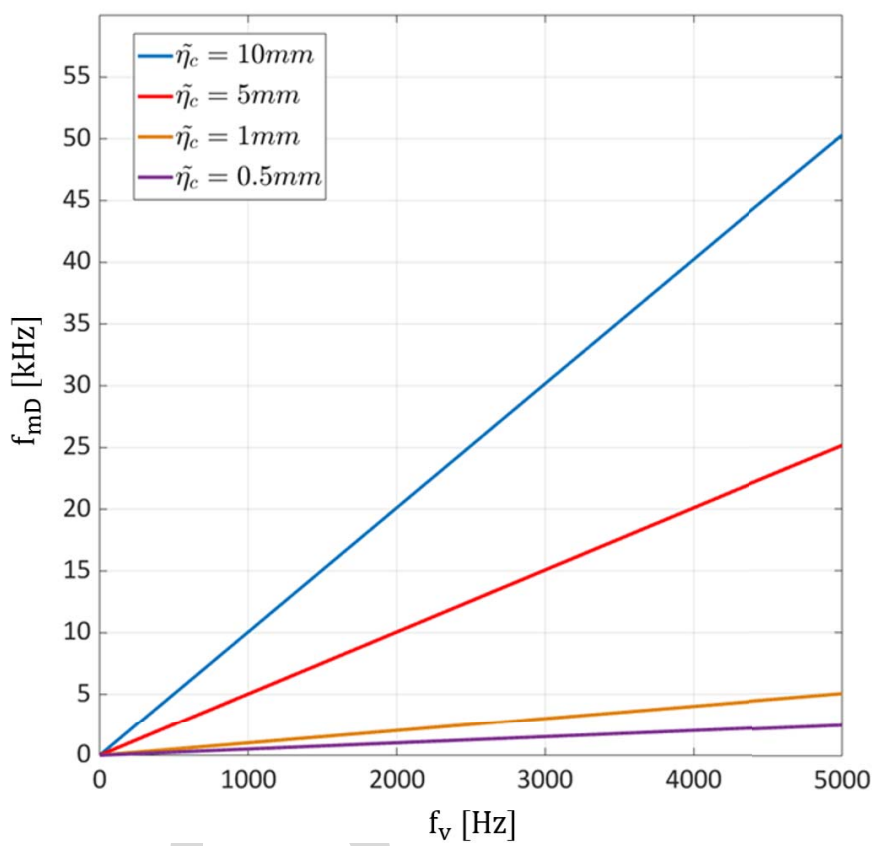

Fig. 8. Theoretical micro Doppler for different displacement $\tilde{\eta}_{c}$ at different vibration frequencies $f_{V}$, with a fixed wavelength $\lambda=1.25 \mathrm{~cm}$.

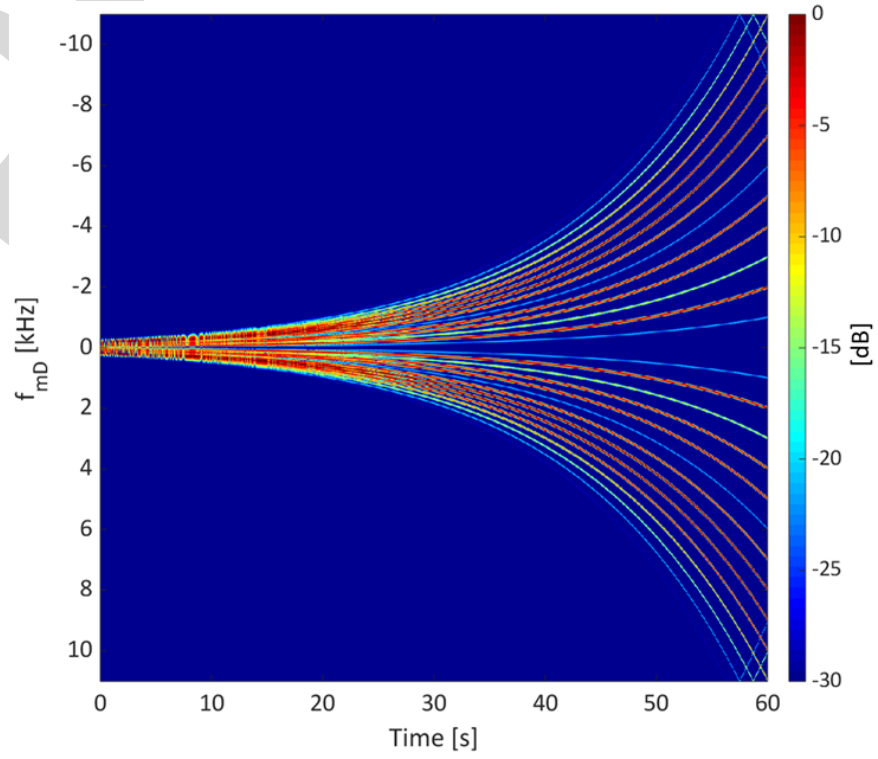

Fig. 9. Magnitude square of the simulated spectrogram of an ideal received radar signal from a speaker playing a $T=60$ seconds exponential sine sweep with $f_{V} \in[20,1000] \mathrm{Hz}$, with fixed displacement equal $\tilde{\eta}_{c}=10 \mathrm{~mm}$

Considering a fixed displacement $\tilde{\eta}_{c}=10 \mathrm{~mm}$ with an exponential chirp of $T=60$ seconds long in the frequency band $f_{v} \in[20,1000] \mathrm{Hz}$, the theoretical maximum micro-Doppler shift achievable is $10 \mathrm{kHz}$. The spectrogram of the simulated received radar signal is shown in Figure 9.

The sinusoidal like motion of the micro Doppler is visible at low vibration frequency. Due to high velocity of the target at high vibration frequency, only the maximum Doppler shift is visible. Furthermore, at high vibration frequency, it is easier to distinguish different harmonics components of the stimulus. 


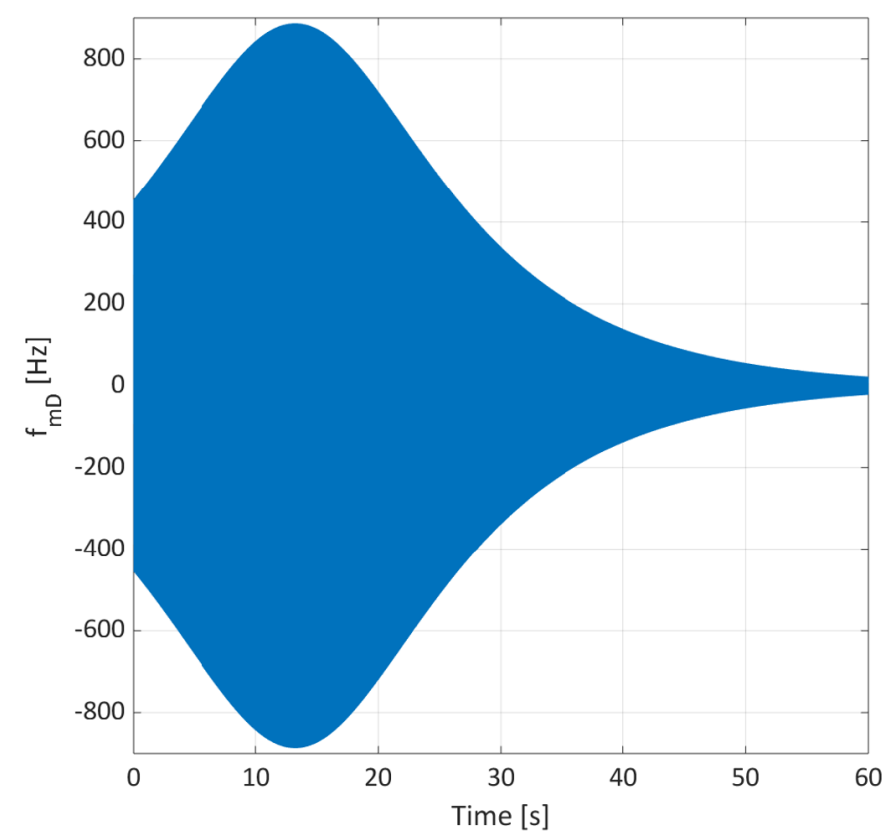

Fig. 10. Theoretical micro-Doppler frequency shift from of a speaker playing an exponential sine sweep of $T=60 \mathrm{~s}$, with $f_{V} \in[20,5000] \mathrm{Hz}$, and initial displacement $\tilde{\eta}_{c}(t=0)=2.26 \mathrm{~cm}$ and $f_{V}(t=0)=20 \mathrm{~Hz}$.
In a more realist scenario the voice coil, modelled as in (1), can be considered constant before the resonance frequency, while after it decreases as the square of vibration frequency $f_{v}$. Then, it is necessary consider both displacement and vibration frequency as function of the time. With this assumption the theoretical micro Doppler equation will become the sum of two components, namely:

$$
f_{m D}(t)=\frac{2}{\lambda} \frac{d \tilde{\eta}_{c}(t)}{d t} x(t)+\frac{4 \pi f_{v}(t)}{\lambda} \tilde{\eta}_{c}(t) \frac{d x(t)}{d t}
$$

Let's consider a loudspeaker with resonance frequency $f_{r}=67.50 \mathrm{~Hz}$, playing an exponential sine sweep of length $T=60$ seconds, with instantaneous vibration frequency $f_{v} \in[20,5000] \mathrm{Hz}$. In the hypotheses of initial displacement $\tilde{\eta}_{c}(t=0)=2.26 \mathrm{~cm}$, related to initial vibration frequency $f_{v}(t=0)=20 \mathrm{~Hz}$, the theoretical micro Doppler frequency is computed by equation (15) and shown in Figure 10.

Unlike the constant displacement scenario, the micro Doppler frequency achieves its maximum value $f_{m D}=887 \mathrm{~Hz}$ at the time instant $t_{\max }=13.2305 \mathrm{~s}$, namely the instant which the vibration frequency $f_{v}$ matches the resonance frequency $f_{r}$ of the speaker itself. As expected, this suggests that the highest micro Doppler shift is achieved at the highest velocity of the speaker, namely at the resonance frequency. Notice that at high vibration frequency, the micro Doppler tends towards zero due to the displacement function. The spectrogram of the simulated received radar signal has shown in Figure 11, where a Blackman-Harris window of $46.5 \mathrm{~ms}$ is used.

From Figure 11 the behaviour of the micro Doppler frequency is confirmed. While the sinusoidal like motion of the micro Doppler is still visible at low vibration frequency achieving the maximum value at the resonance frequency, at high vibration frequency it is clear the strong component

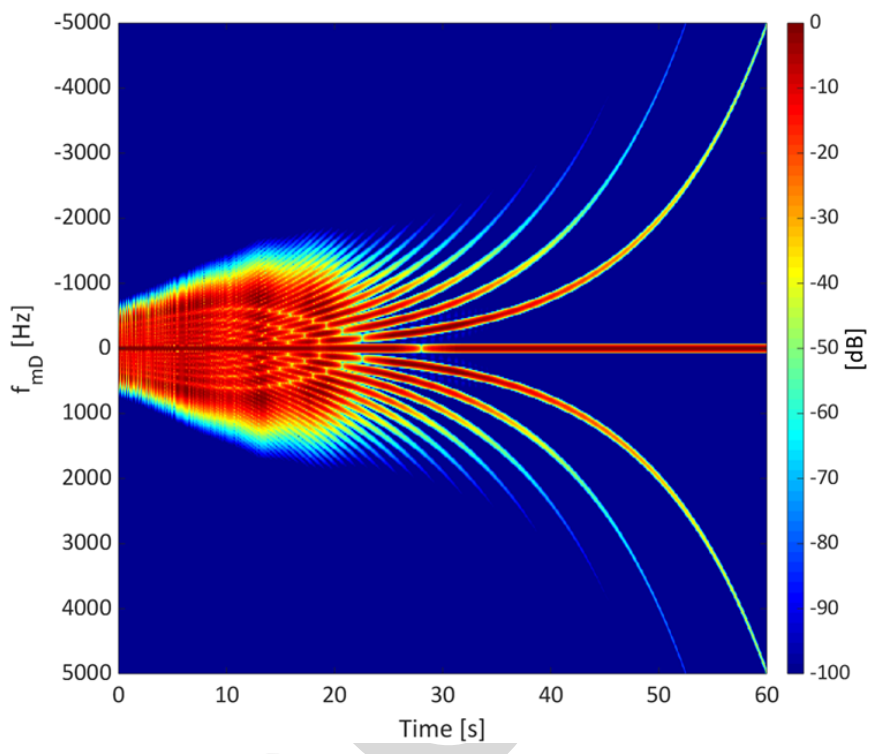

Fig. 11. Magnitude square of the spectrogram of the simulated received radar signal from a speaker playing an exponential sine sweep of $T=60 \mathrm{~s}$, with $f_{V} \in[20,5000] \mathrm{Hz}$, and initial displacement $\tilde{\eta}_{c}(t=0)=$ $2.26 \mathrm{~cm}$ and $f_{V}(t=0)=20 \mathrm{~Hz}$.

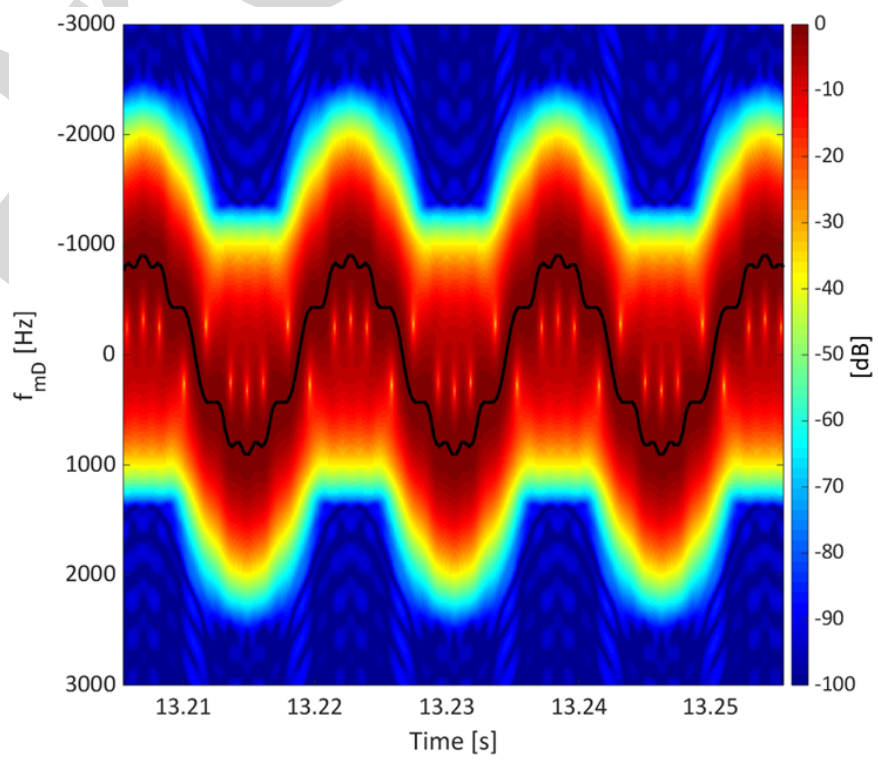

Fig. 12. Magnitude square of the spectrogram of time window of $0.05 \mathrm{~s}$ of the simulated received radar signal around the time instant $t_{\max }$, where Doppler shift achieves its maximum, namely in proximity of the resonance frequency $f_{r}$ of the speaker. The maximum Doppler shift is highlighted with a black line.

at the zero frequency. The spectrogram of a time window of $0.05 \mathrm{~s}$ of the simulated received radar signal around $t_{\max }$ is shown in Figure 12, confirming the sinusoidal like motion of the micro Doppler.

Due to the fast vibration of the speaker on the Line of Sight (LOS), a phenomenon known as coupled echoes will appear [5]. The result of this effect will be the presence of "ghost returns" in the Doppler direction on both sides of the original target. So the speaker vibration can then introduce an infinite series of paired echoes $m$ because, when 
considering (15), the received signal $s_{r}$ in (13) may be expressed as a series of expansion of Bessel functions of the first kind of order $m$ :

$$
J_{m}\left(\frac{4 \pi \tilde{\eta}_{c}}{\lambda}\right)=\frac{1}{2 \pi} \int_{-\pi}^{+\pi} \exp \left\{j\left(\frac{4 \pi \tilde{\eta}_{c}}{\lambda} \sin (u)-m u\right)\right\} d u
$$

such that the received radar signal $s_{r}$ in baseband can be expressed as:

$$
\begin{aligned}
s_{r}(t)= & \rho \exp \left\{j \frac{4 \pi R_{0}}{\lambda}\right\} \exp \left\{j 2 \pi f_{0} t\right\} \\
& \times \sum_{m=-\infty}^{+\infty} J_{m}\left(\frac{4 \pi \tilde{\eta}_{c}}{\lambda}\right) \exp \left\{m j \frac{2 \pi f_{1}\left(k^{t}-1\right)}{\log k}\right\} \\
= & \rho \exp \left\{j \frac{4 \pi R_{0}}{\lambda}\right\} \exp \left\{j 2 \pi f_{0} t\right\}\left\{J_{0}\left(\frac{4 \pi \tilde{\eta}_{c}}{\lambda}\right)\right. \\
& +J_{1}\left(\frac{4 \pi \tilde{\eta}_{c}}{\lambda}\right) \exp \left\{j \frac{2 \pi f_{1}\left(k^{t}-1\right)}{\log k}\right\}+ \\
& -J_{1}\left(\frac{4 \pi \tilde{\eta}_{c}}{\lambda}\right) \exp \left\{-j \frac{2 \pi f_{1}\left(k^{t}-1\right)}{\log k}\right\} \\
& +J_{2}\left(\frac{4 \pi \tilde{\eta}_{c}}{\lambda}\right) \exp \left\{j \frac{4 \pi f_{1}\left(k^{t}-1\right)}{\log k}\right\}+ \\
& \left.-J_{2}\left(\frac{4 \pi \tilde{\eta}_{c}}{\lambda}\right) \exp \left\{-j \frac{4 \pi f_{1}\left(k^{t}-1\right)}{\log k}\right\}+\ldots\right\}
\end{aligned}
$$

with $k$ the exponential chirp rate. Therefore, the micro-Doppler frequency spectrum consists of pairs of spectral lines around the center frequency $f_{0}$ and with spacing $f_{v}$ between adjacent lines. The intensity of paired echoes visible depends from the modulation index $\Upsilon=\beta \tilde{\eta}_{c}$. In case of wideband modulation $(\Upsilon>1)$ more spectral lines appear. This is visible in the spectrograms in Figure 9 where, a fixed displacement of $10 \mathrm{~mm}$ makes the signal wideband modulated with fixed modulation index: multiple and very densely spaced paired echoes are introduced. Due to a non constant displacement, a different behaviour is shown through the spectrogram in figure 11 where the received signal will be wideband modulated at low vibrational frequency and narrowband modulated at high vibrational frequency. Thus, more harmonics are detected at low vibration frequencies. This results is in agreement with loudspeaker modelling theory, where the harmonics components at low vibration frequencies are defined as regular non linear distortions components, generated by the non linear behaviour of stiffness, force factor and inductance of the driver.

\section{Mechanical Characterization of a Speaker}

The ability of the radar technology to detect the motion of a speaker has been described above. When a chirp is used in the simulated scenario, the voltage as the force factor is supposed to be constant with the frequency of the stimulus. In a real scenario this hypothesis is not justified due to the non linearities, introduced for example by stiffness $\left(K_{m s}\left(\tilde{\eta}_{c}\right)\right)$, force factor $\left(B l\left(\tilde{\eta}_{c}\right)\right)$ and inductance $\left(L_{e}\left(\tilde{\eta}_{c}\right)\right)$. To understand the influence of the non linearities on the speaker behaviour, in terms of deviation from the ideal piston mode behaviour, an alternative approach is considered. Commonly used in radar is the matched filter technique, obtained by correlating a known signal with an unknown signal to detect the presence of the template in the unknown signal. This is the radar equivalent of the acoustic measurement technique introduced in [16]-[18], where the unknown signal is convolved with a conjugated time-reversed version of the template. With this technique the speaker can be mechanically characterized. The matched filter is the optimal linear filter for maximizing the signal-to-noise ratio (SNR) in the presence of additive stochastic noise. If the model of the ideal received radar signal is found, matched filter technique could be applied to RF sensors in order to characterize the mechanical behaviour of the speaker. Using (13) the received radar signal in baseband of an ideal loudspeaker, behaving as piston mode in the full frequency band, is described. It can be seen as the product between the magnitude and phase components. While the phase component depends on both $T \& S$ parameters of the speaker and the stimulus waveform, the magnitude component introduces uncertainty since it is an estimation of the target reflectivity, which is usually difficult to estimate. For this reason, to reduced the amount of uncertainty, the system's impulse response can be computed by simply correlating the phase of the measured radar signal $y(t)$ with the phase of the simulated signal $s_{r}(t)$, such that:

$$
h(t)=\angle y(t) \star \angle s_{r}(t) .
$$

where $\star$ is the correlation operator. With an exponential sine sweep of $T=60$ seconds long as a test signal, and with the hypothesis of a linear system, the results would be a perfect peak centred in $T$, defined as linear impulse response, as shown in the Figure 13.

In a real scenario instead, where the Device Under Test (DUT) is never linear, along with the linear impulse responses, non linear impulse responses are also obtained, corresponding to the various harmonics of the input signal. With the exponential sine sweep, these non linear product, do not contaminate the linear impulse response, as they are occurring at very precise anticipatory times $\Delta t$ before the linear response, namely:

$$
\Delta t=T \frac{\ln (N)}{\ln \left(\frac{f_{2}}{f_{1}}\right)}
$$

where $N$ is the Nth distortion component. Thus, the signal component of the time waveform at the output of the matched filter is actually the autocorrelation function $r_{S_{r}, s_{r}}$ of the ideal signal. The matched filter peak $h(T)$ is then $r_{s_{r}, s_{r}}(0)=E_{s_{r}}$, where $E_{s_{r}}$ is the total energy in the signal $s_{r}(t)$ [19]. Applying a window around the peak $h(T)$, it is possible to compute the linear frequency response through Fourier Transform. In the event where no window is applied, the Power Spectral Density (PSD) of the signal is computed, where the harmonic components and noise are incorporated into the frequency response. In Figure 14 the PSD of the simulated radar signal from an ideal speaker is shown.

Later in this paper, the harmonic distortion responses will not be discarded but analysed. The system's response is 


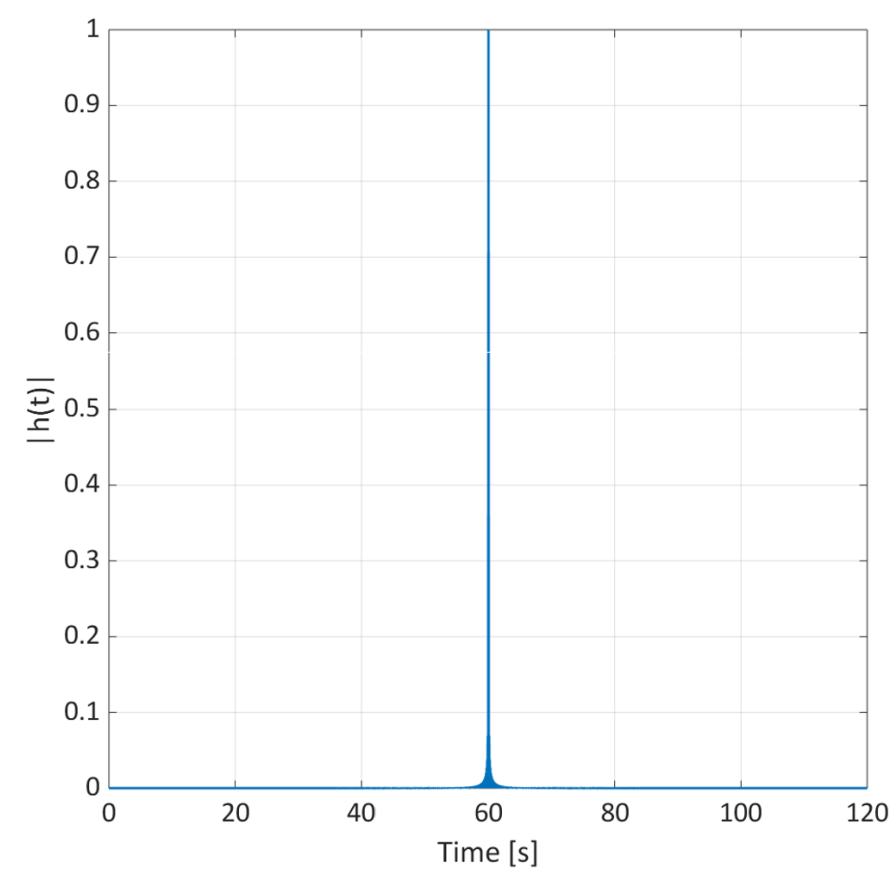

Fig. 13. Matched filter output of the simulated radar signal from an ideal speaker, playing an exponential sine sweep of $T=60$ s with $f_{V} \in[20,5000] \mathrm{Hz}$.

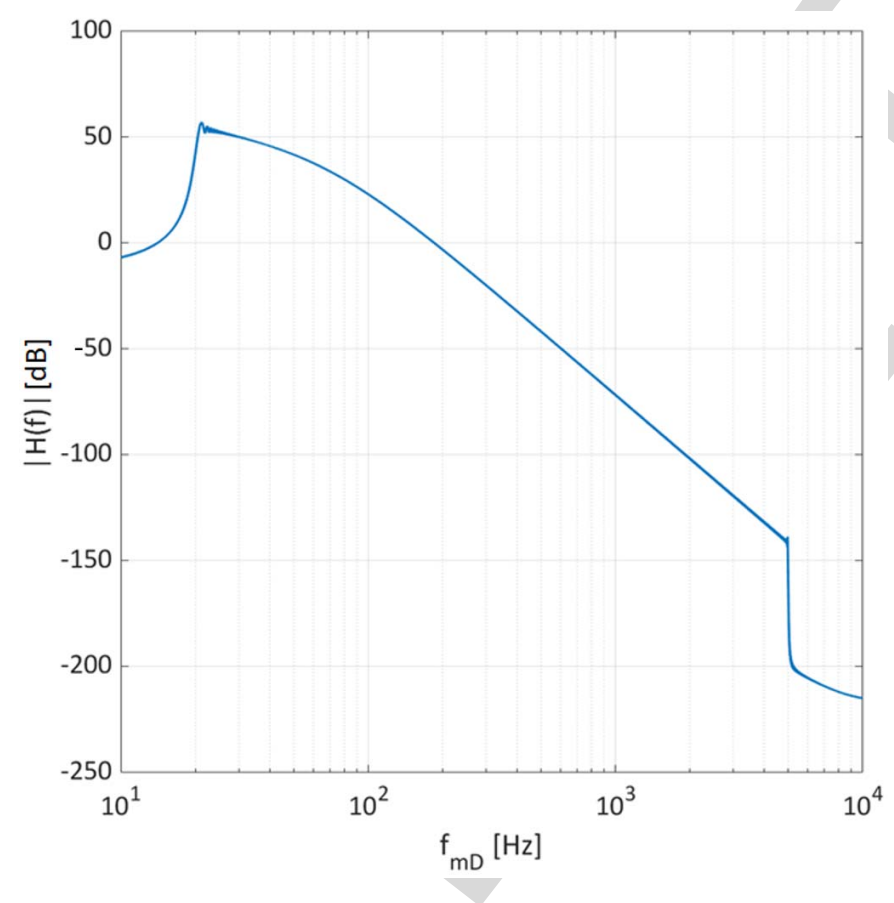

Fig. 14. Normalised frequency responses of an ideal speaker, playing a exponential sine sweep of $T=60 \mathrm{~s}$ with $f_{V} \in[20,5000] \mathrm{Hz}$. affected in varying ways by different irregular defects, making the non-linear behaviour of the loudspeaker vibrations a powerful indicator of possible manufacturing problems. Thus, in the real scenario it is possible to define the time waveform at the output of the matched filter as the cross correlation function $r_{y, s_{r}}$ between the measured signal $y(t)$ and the ideal

TABLE I

Measured Thiele\&Small Parameters of B\&C $10 C L 51$ LF Driver

\begin{tabular}{cc}
\hline Parameter & Value \\
\hline$f_{r}$ & $67.57 \mathrm{~Hz}$ \\
$B l$ & $9.67 \mathrm{~N} / A$ \\
$Q_{t s}$ & 0.5425 \\
$Q_{e s}$ & 0.6075 \\
\hline
\end{tabular}

one $s_{r}(t)$, where its Fourier Transform is referred as Cross Power Spectrum Density (CPSD).

525 526

\section{Real Measurement Analysis}

In this section, real data acquisitions are analysed and compared with simulation results. In Section IV-A, the microDoppler signature is analysed considering a single tone acoustic signal. The micro-Doppler signature of a speaker playing a sine sweep is analysed in Section IV-B. Finally, in Section IV-C the mechanical characterization of a real speaker is shown. For all of the analyses, the signal amplitude was set to $-6 \mathrm{~dB}$ for the standard "Loudness units relative to Full Scale" (LUFS) to prevent any digital or analog clipping in the measurement chain. In order to simulate a received radar signal, a diameter of $25 \mathrm{~cm}$ (which is a typical dimension for a loudspeaker operating in this frequency range) has been considered. The backscattering coefficient $\rho$ [5] coming from the only vibrating metallic component, namely the voice coil, is calculated as in (10), with the radius of voice coil $r=2.55 \mathrm{~cm}$. The measurements acquisition was conducted through a bespoke $24 \mathrm{GHz} \mathrm{CW}$ radar made by WhiteHorse Radar LTD. It has been used to measure the returns from a $25 \mathrm{~cm}$ low frequency driver placed $1 \mathrm{~m}$ away from the radar on the Line Of Sight (LOS). For both simulated and real data, a sampling frequency $f_{s}=22 \mathrm{kHz}$ is considered. Through a Clio Pocket board [20], the electromechanical parameters needed to feed the model of the ideal received radar signal are computed. The measured T\&S parameters of $B \& C 10 C L 51 \mathrm{LF}$ driver [21] are reported in Table I.

In Figure 15, the system set up is shown. The input signal to the loudspeaker has been generated by Adobe Audition 3.0, while the received signal is acquired by the radar through Matlab R2018a, also used to process the data.

\section{A. Micro Doppler Signature: Single Tone Analysis}

In this section, the single tone analysis is performed, where real data are analysed and compared to simulation results. A single tone has been chosen as acoustic input to the loudspeaker with frequency $f_{v}=67 \mathrm{~Hz}$ to drive an ideally flat and rigid disk behaving in piston mode, at its resonance frequency. To understand the ability of the radar to detect the motion of the speaker, two different output voltage were be taken in consideration. Setting the voltage at the loudspeaker terminals to be $5 \mathrm{~V}$, the normalized spectrum of the received signal is shown in Figure 16.

Having the signal in baseband, in the spectrum in Figure 16 the positive frequency band is referred as positive direction 


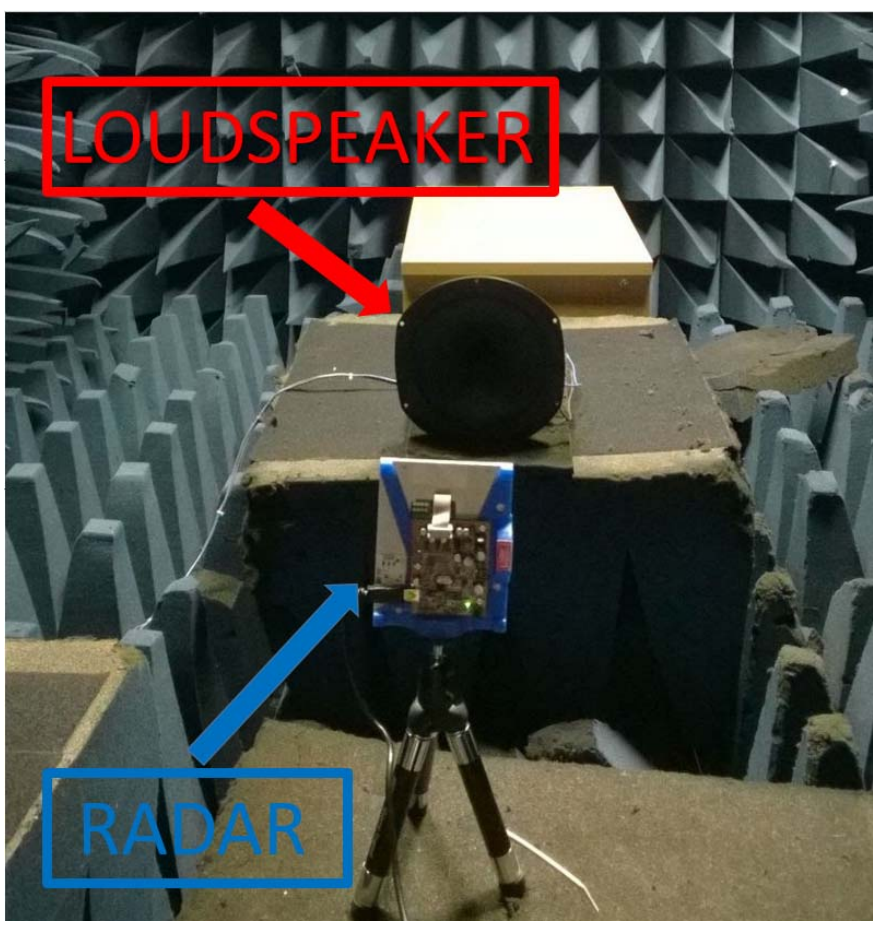

Fig. 15. Experiment setup.

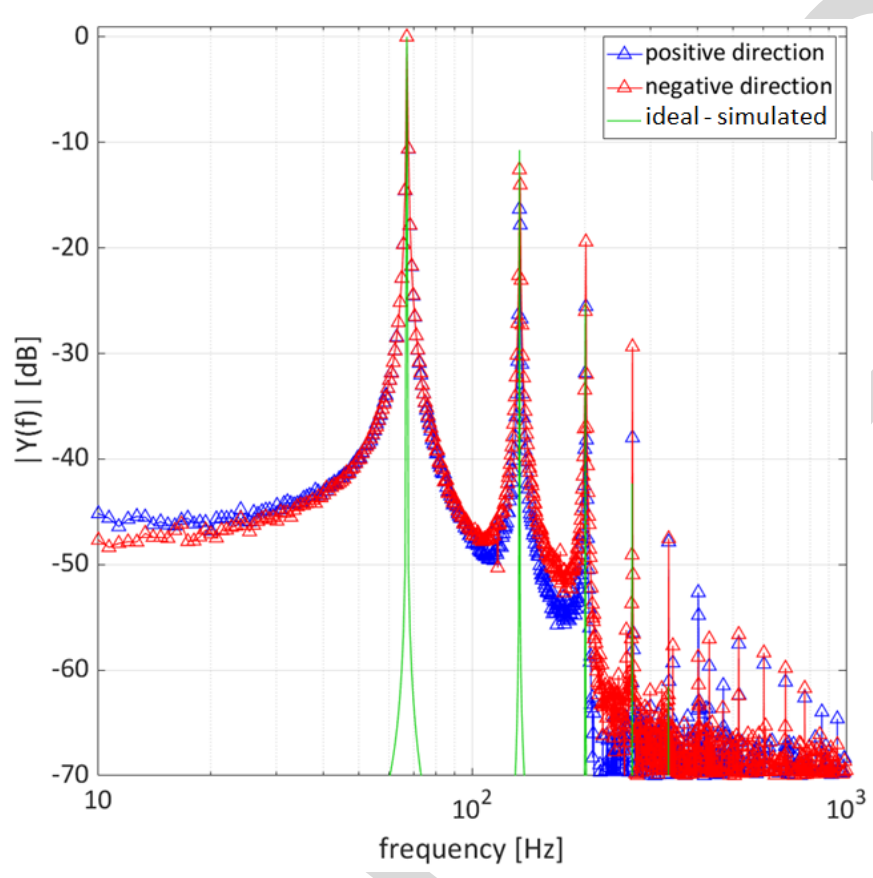

Fig. 16. Spectrum of real radar measurement from a $25 \mathrm{~cm}$ loudspeaker playing a single tone $f_{V}=67 \mathrm{~Hz}$ at its resonance frequency at $5 \mathrm{~V}$ output voltage. while the negative as negative displacement. By Fourier analysis the vibration frequency of the coil is detected correctly. Although the presence of the noise floor, the fundamental component and its harmonics are visible and in agreement with the spectrum of the simulated signal. The discrepancy between the ideal and the real spectrum is related to the non linear effect of the DUT, previously defined. Moreover, the discrepancy

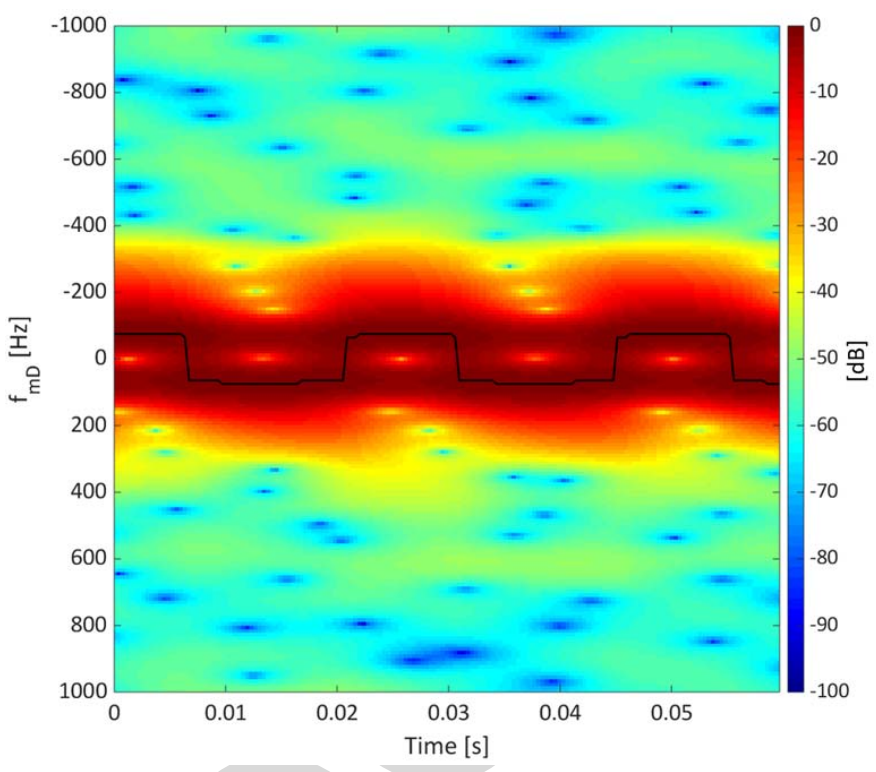

Fig. 17. Magnitude square of the spectrogram of real radar measurement from a $25 \mathrm{~cm}$ loudspeaker playing $f_{V}=67 \mathrm{~Hz}$ single tone at $5 \mathrm{~V}$ output voltage, with Blackman-Harris window of $23.2 \mathrm{~ms}$. The maximum Doppler shift is highlighted with a black line.

between the positive (blue curve) and negative direction (red curve) may be related to the effect of non linear stiffness. In Figure 17, a Blackman-Harris window of $23.2 \mathrm{~ms}$ long is used to generate the spectrogram of the radar signal.

From the spectrogram the maximum frequency Doppler shift can be evaluated and from this the displacement. Inverting (11), the maximum value of $\tilde{\eta}_{c, 5 V}$ can be obtained. As in the simulated scenario (in Figure 6(b)), the micro Doppler has a maximum value equal to $75 \mathrm{~Hz}$, in both positive and negative direction, leading to the estimation of the displacement equal to $1.1 \mathrm{~mm}$. In the case of an output voltage of $10 \mathrm{~V}$, the speaker should be more prone to distortion. This is visible from the spectrum shown in Figure 18, where harmonics with higher magnitude appear due to a larger displacement are visible from the spectrogram in Figure 19.

Although the behaviour is still in agreement with the model in Figure 7, some discrepancies appear. The differences with the ideal micro Doppler are illustrated in Figure 20.

While in the simulated scenario the micro Doppler profile has a maximum and minimum value equal to $150 \mathrm{~Hz}$, the measured one resulted to be $150 \mathrm{~Hz}$ in the positive direction and $172 \mathrm{~Hz}$ in the negative direction. This suggests that, due to non linear effects, the voice coil is susceptible to acceleration in order to reach the farthest point from the radar, visible through the different rising and falling front from the simulated one. This can be confirmed by the phase of the signal.

In Figure 21 and 22 the phase of the real and simulated radar signals are compared when an output voltage is set to $5 \mathrm{~V}$ and $10 \mathrm{~V}$, respectively. It can be seen that the phase of the real data matches the simulated one in terms of sinusoidal-like motion, especially when the applied voltage is $5 \mathrm{~V}$. However discrepancies between the simulated and real data appear at $10 \mathrm{~V}$ of applied voltage. Comparing the rising and falling front of the measured phase in Figure 22 with the corresponding 


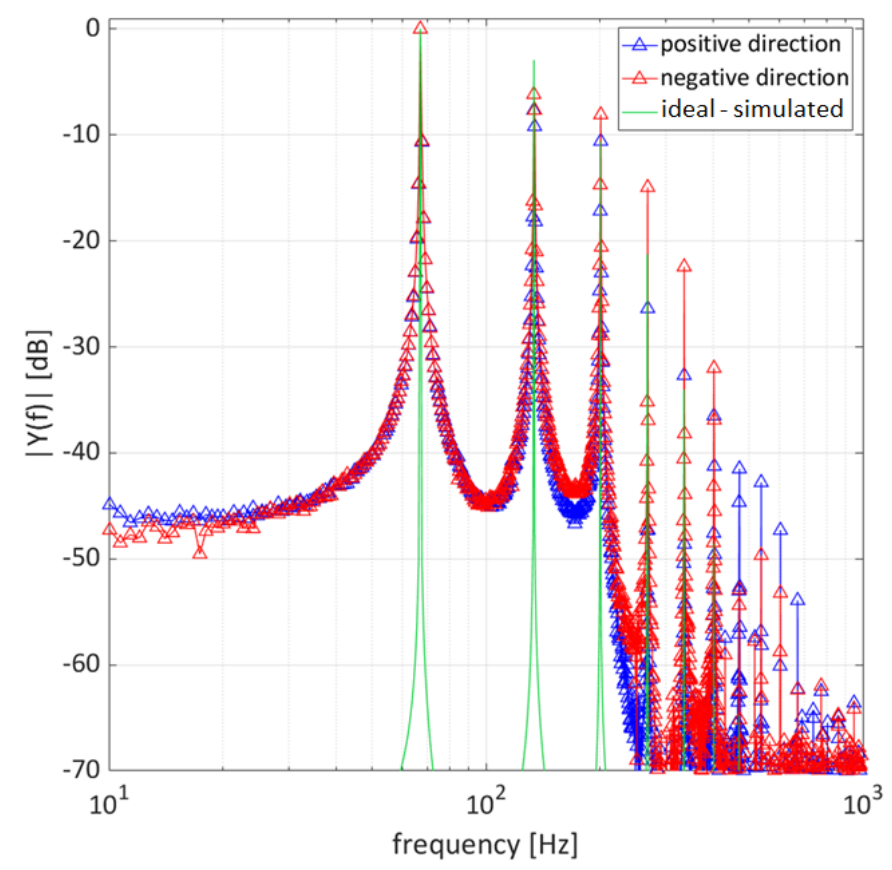

Fig. 18. Spectrum of real radar measurement from a $25 \mathrm{~cm}$ loudspeaker playing a single tone $f_{V}=67 \mathrm{~Hz}$ at its resonance frequency at $10 \mathrm{~V}$ output voltage.

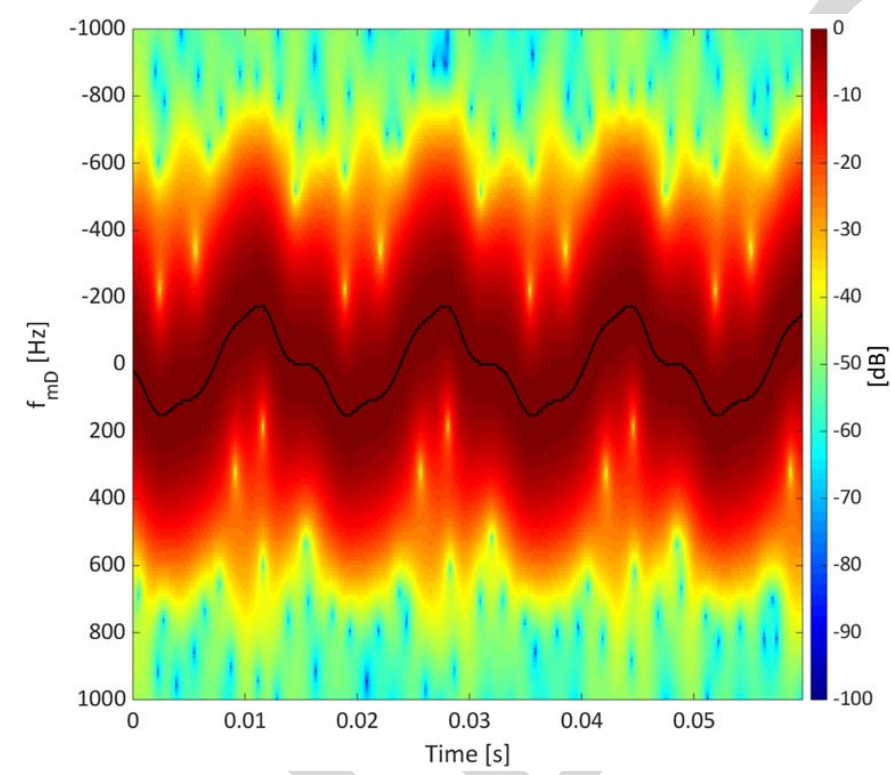

Fig. 19. Magnitude square of the spectrogram of real radar measurement from a $25 \mathrm{~cm}$ loudspeaker playing $f_{V}=67 \mathrm{~Hz}$ single tone at $10 \mathrm{~V}$ output voltage, with Blackman-Harris window of $5.8 \mathrm{~ms}$. The maximum Doppler shift is highlighted with a black line. simulated one, it is possible to observe that actual motion does not match completely the ideal one. From the plot it is possible to infer that the voice coil spends more time in the position further away from the radar than the piston model suggests. A possible explanation of this phenomenon could be the superposition of two components. The first is the non linearity introduced by the stiffness. Loudspeakers use a suspension system to center the coil in gap and to generate a

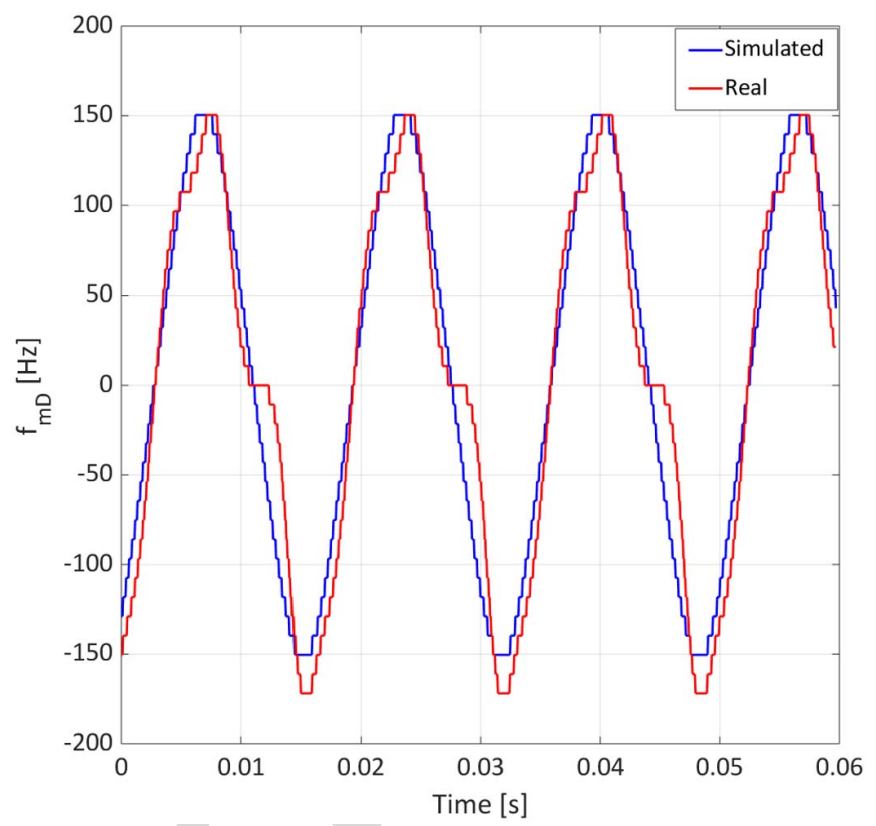

Fig. 20. Micro Doppler comparison between the simulated signal and the real radar measurement from a $25 \mathrm{~cm}$ loudspeaker playing $67 \mathrm{~Hz}$ single tone at $10 \mathrm{~V}$ output voltage, with Blackman-Harris window of $5.8 \mathrm{~ms}$.

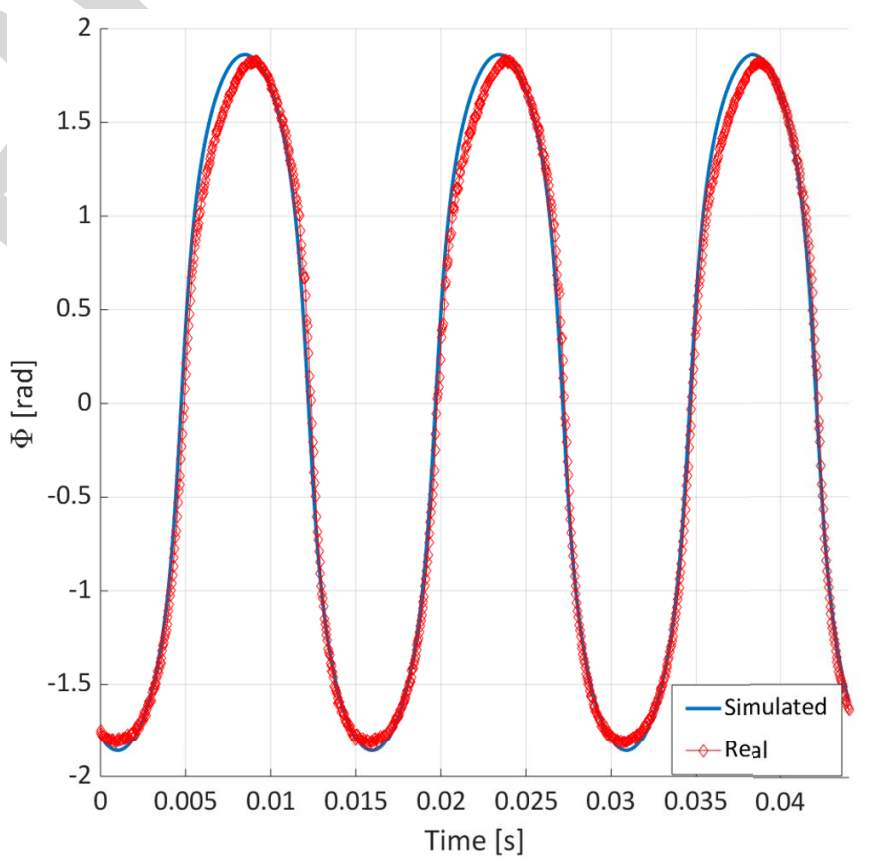

Fig. 21. Comparison between the phase of the simulated and real radar signal of the speaker playing tone $f_{V}=67 \mathrm{~Hz}$ equal at its resonance frequency $f_{r}$, with output voltage set to $5 \mathrm{~V}$.

restoring force which moves the coil back to the rest position. Thus, spider and surround behave like a normal spring: at low displacement there is an almost linear relationship, while at high displacement the suspension responds with more force than the predicted one. The second component could derive from the non linearity introduced by the force generated by the magnetic field times the length of the voice coil immersed in the gap: if the coil windings leave the gap, the force factor 


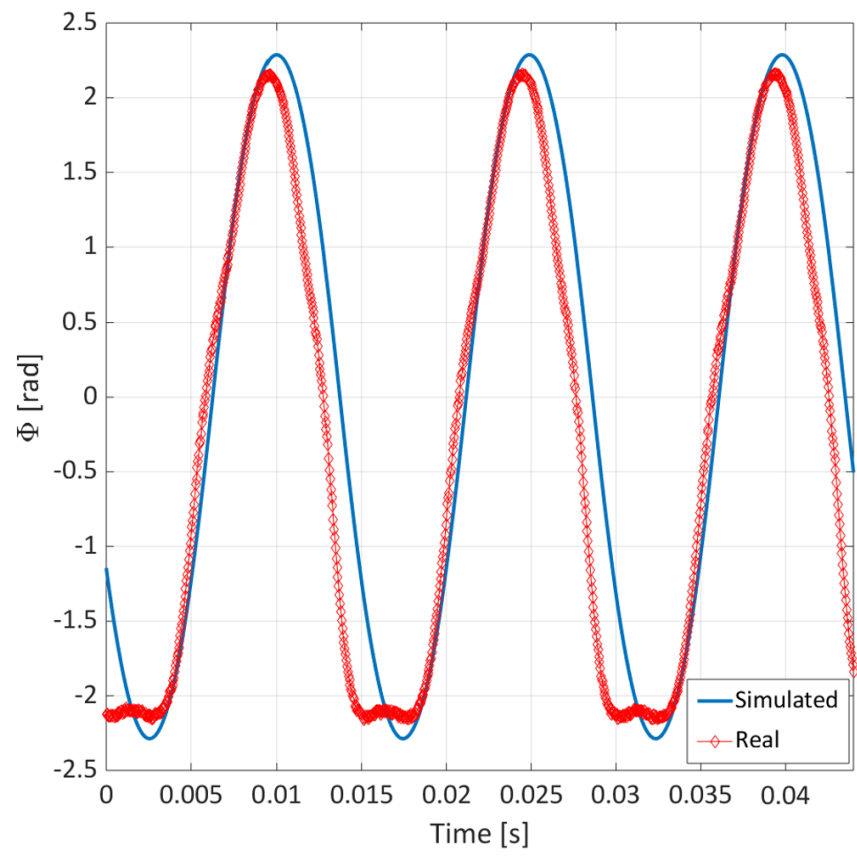

Fig. 22. Comparison between the phase of the simulated and real radar signal of the speaker playing tone $f_{V}=67 \mathrm{~Hz}$ equal at its resonance frequency $f_{r}$, with output voltage set to $10 \mathrm{~V}$.

decreases [3]. Consequently, the non linearities appear and the coil is pushed back by magnetic field, from the nearest to the furthest position from the radar, earlier than its ideal one.

\section{B. Micro Doppler Signature: Sine Sweep Analysis}

In this section the micro Doppler signature of a speaker playing a sine sweep will be analysed and compared to the simulation results. The acoustic tone varies from a starting frequency $f_{1}=20 \mathrm{~Hz}$ at time $t=0$, ending at the time $T=60 \mathrm{~s}$ with frequency $f_{2}=5 \mathrm{kHz}$. The speaker was connected to an amplifier, which output was set to $\tilde{e}_{g}=3 \mathrm{~V}$ at $1 \mathrm{kHz}$. With the speaker parameters in Table I, the theoretical displacement is modelled through (1): at time $t=0$ the maximum value of $\tilde{\eta}_{c}=1.1 \mathrm{~mm}$ is found. The spectrograms of both simulated and real radar signal are compared and shown in Figure 23. Both the spectrograms are produced using a Blackman-Harris window of $46.5 \mathrm{~ms}$, with an overlap of $99 \%$, with column based normalization.

The first difference that can be immediately noted, it is the presence of the noise floor. While the simulated signal has been generated in absence of noise, the real one shows a background noise increasing with time. This result is in agreement with radar sensitivity, shown in Table II: as a vibration amplitude of a micron results in a phase shift of only $0.06 \mathrm{deg}$, it is almost undetectable.

It can be seen in Figure 23b that a high intensity distortion is visible at the time instant $t=42 \mathrm{~s}$, with vibration frequency $f_{v}$ approximately $1 \mathrm{KHz}$. Due to rocking modes, DC displacement and motor instability, the speaker deviates from the "piston mode," making voice coil rubbing and hard bottoming typical defects. From the radar point of view this effect can be explained through the concept of

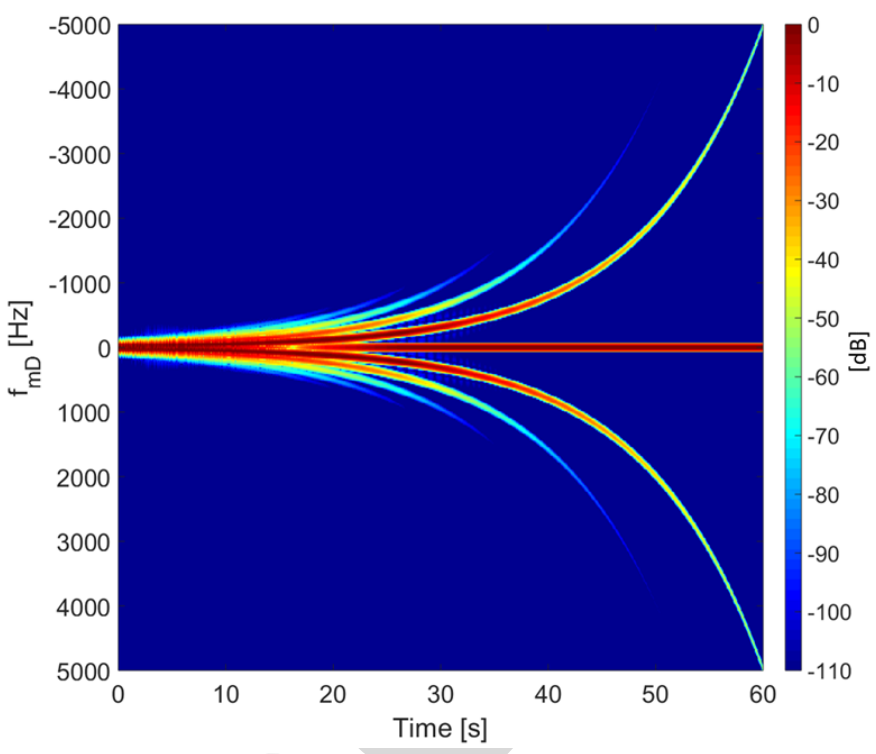

(a) Spectrogram Simulated Signal

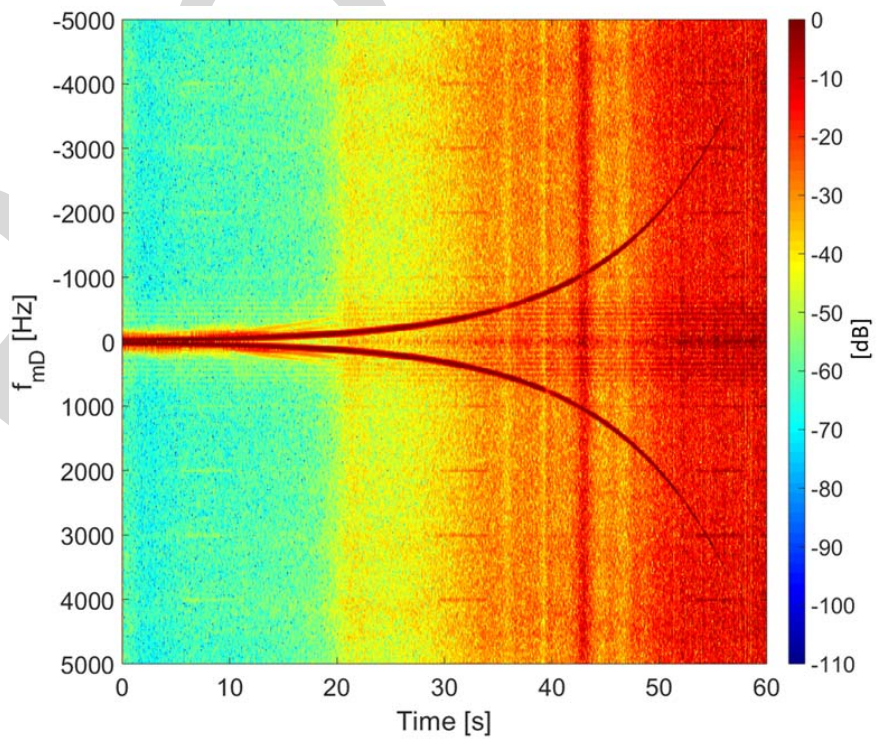

(b) Spectrogram Real Signal

Fig. 23. Magnitude square of the spectrogram of simulated and real received radar signal from a speaker playing an exponential chirp, with T\&S parameter of Table I, with Blackman-Harris window of $46.5 \mathrm{~ms}$.

TABLE II

SENSITIVITY OF A 24GHz CW RADAR TO DifFERENT VIBRATION AMPLITUDE

\begin{tabular}{cc}
\hline Vibration Amplitude & Maximum Phase Shift \\
\hline $1 \mathrm{~cm}$ & $576 \mathrm{deg}$ \\
$1 \mathrm{~mm}$ & $58 \mathrm{deg}$ \\
$1 \mu \mathrm{m}$ & $0.06 \mathrm{deg}$ \\
\hline
\end{tabular}

disruptive interference. Whenever waves originating from two or more sources interact with each other, there will be phasing effects leading to an increase or decrease in wave energy at the point of combination. When elastic waves of the same frequency meet in such a way that their displacements are precisely synchronized (in phase, or 0 degree phase angle), 


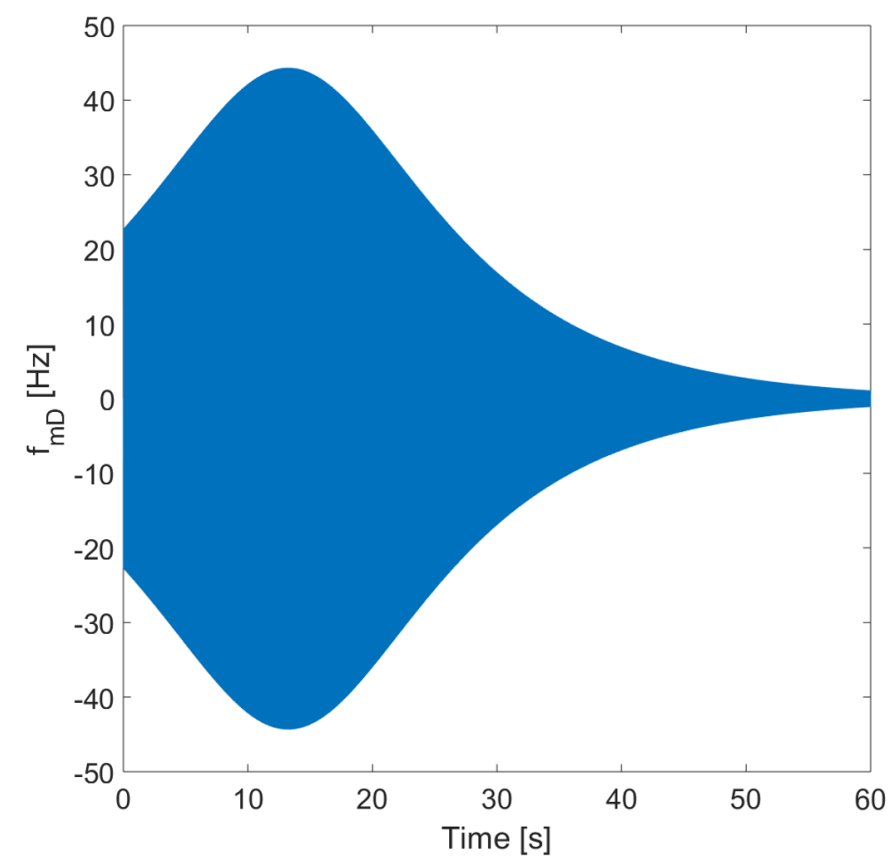

Fig. 24. Theoretical micro Doppler frequency shift of a speaker playing a 60 seconds exponential sine sweep with $f_{V} \in[20,5000] \mathrm{Hz}$, with T\&S parameter of Table I. the wave energies will add together to create a larger amplitude wave. If they meet in such a way that their displacements are exactly opposite (180 degrees out of phase), then the wave energies will cancel each other. At phase angles between 0 degrees and 180 degrees, there will be a range of intermediate stages between full addition and full cancellation. Using the mathematical formulation in (15), the theoretical micro Doppler related to the theoretical displacement is shown in Figure 24.

Also in this case the maximum Doppler shift happens at the resonance frequency of the speaker, with maximum value of $f_{m D_{M A X}}=44.3 \mathrm{~Hz}$ at the time $t_{\max }=13.23 \mathrm{~s}$. The spectrograms of both simulated and real radar signal around $t_{\max }$ are shown in Figure 25.

From the spectrograms in Figure 25 is possible to appreciate how the simulated signal matches with the real measurement. In both the spectrograms, a maximum Doppler shift of $107 \mathrm{~Hz}$ is detected with a Blackman-Harris window of $11.6 \mathrm{~ms}$. Due to both the spectrogram time-frequency dilemma and coupled echoes phenomenon, the maximum Doppler shift detected differs from the theoretical one making the echoes stronger than the main component. Thus, for a correct characterization of the speaker, an alternative approach is needed and introduced in the next section.

\section{Mechanical Characterization of a Speaker}

With the displacement model introduced in the section II, the performance of the loudspeaker at low frequency can be estimated. This estimation is computed considering small input signal levels for which the mechanical behaviour of the driver is effectively linear. In order to understand the effects introduced by the non linear components, the matched filter

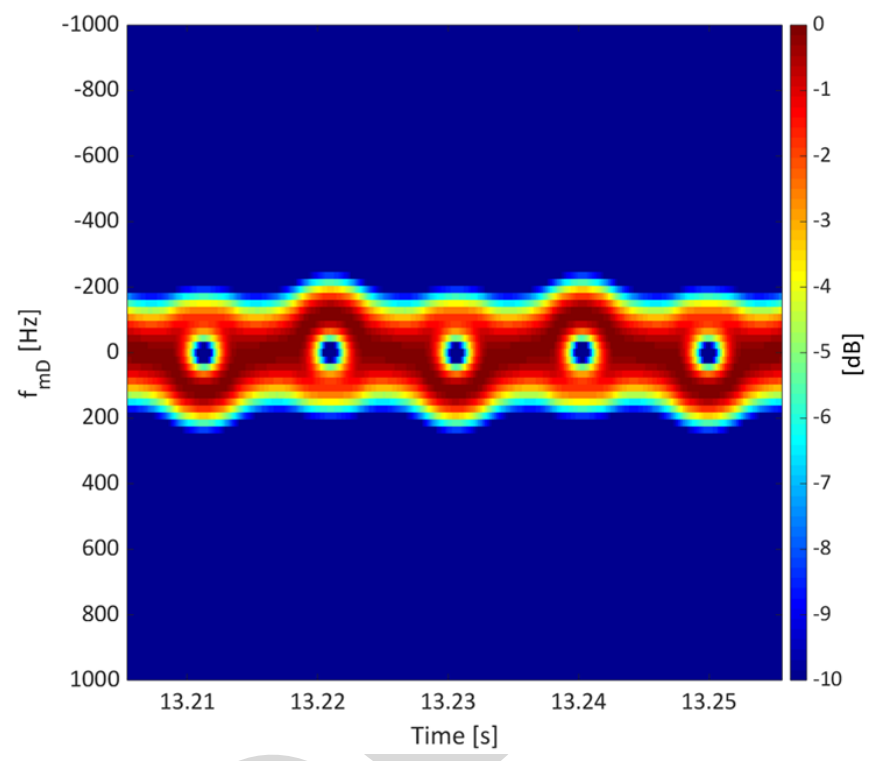

(a) Spectrogram Simulated Signal

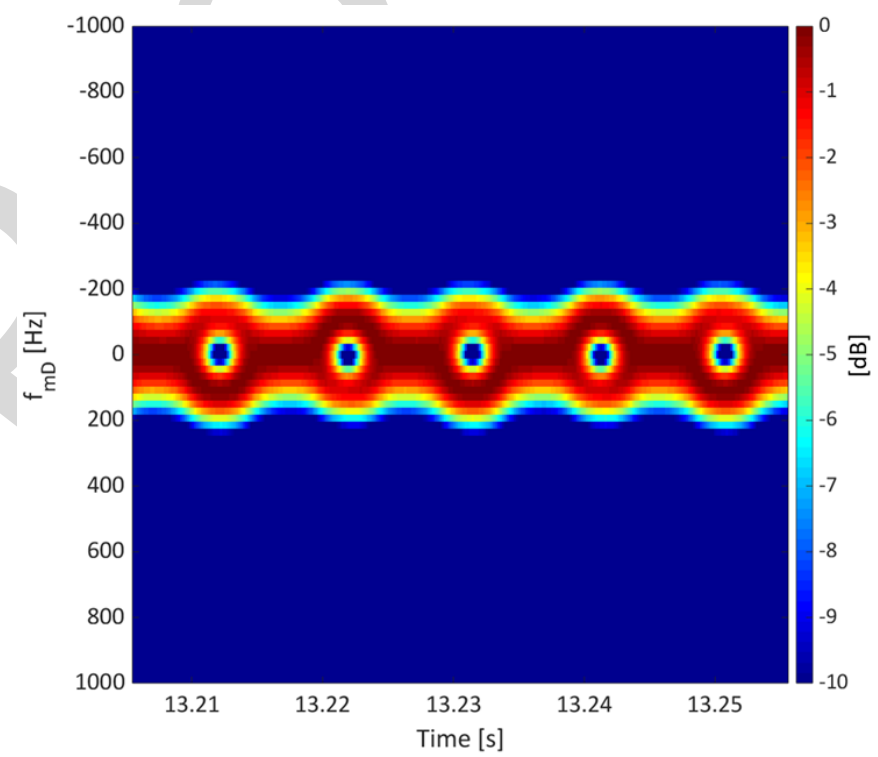

(b) Spectrogram Real Signal

Fig. 25. Magnitude square of the spectrogram of simulated and real received radar signal from a speaker playing an exponential chirp, with T\&S parameter of Table I, at the $t=t_{\max }$ and $f_{V}=f_{r}$, with Blackman-Harris window of $11.6 \mathrm{~ms}$.

approach is used. In case of perfect linear system, the matched filter output would consist in a perfect peak centred at the instant $T$ equal to the length of the test signal, defined as linear impulse response. Real devices unfortunately are never linear; thus, not only a linear impulse response appears, but also non linear impulse responses are obtained, corresponding to the various harmonics of the input signal. This is visible in the Figure 26, where the matched filter is applied to a real measurement.

In agreement with (19), the non linear products occur at very precise anticipatory time before the linear response, namely at $\Delta t_{2 n d}=7.50 \mathrm{~s}, \Delta t_{3 r d}=11.93 \mathrm{~s}$ and $\Delta t_{4 t h}=15.04 \mathrm{~s}$. Applying a Fourier Transform to the matched filter output, the frequency 


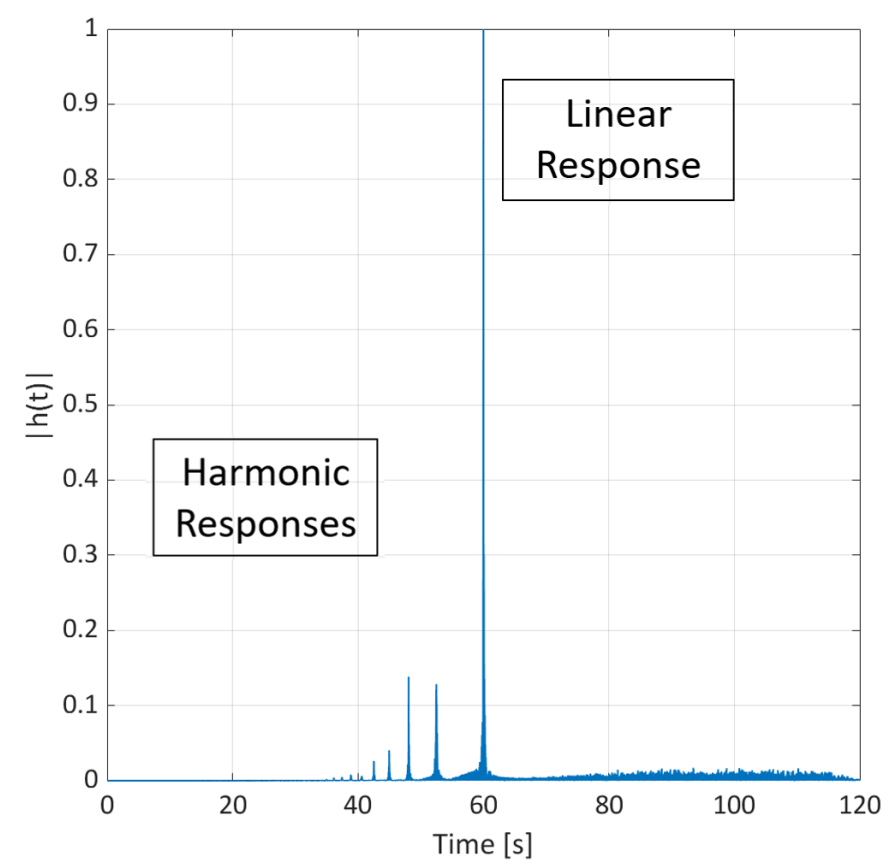

Fig. 26. Matched filter output: linear and non linear impulse responses of the DUT, with measured T\&S parameters of table I.

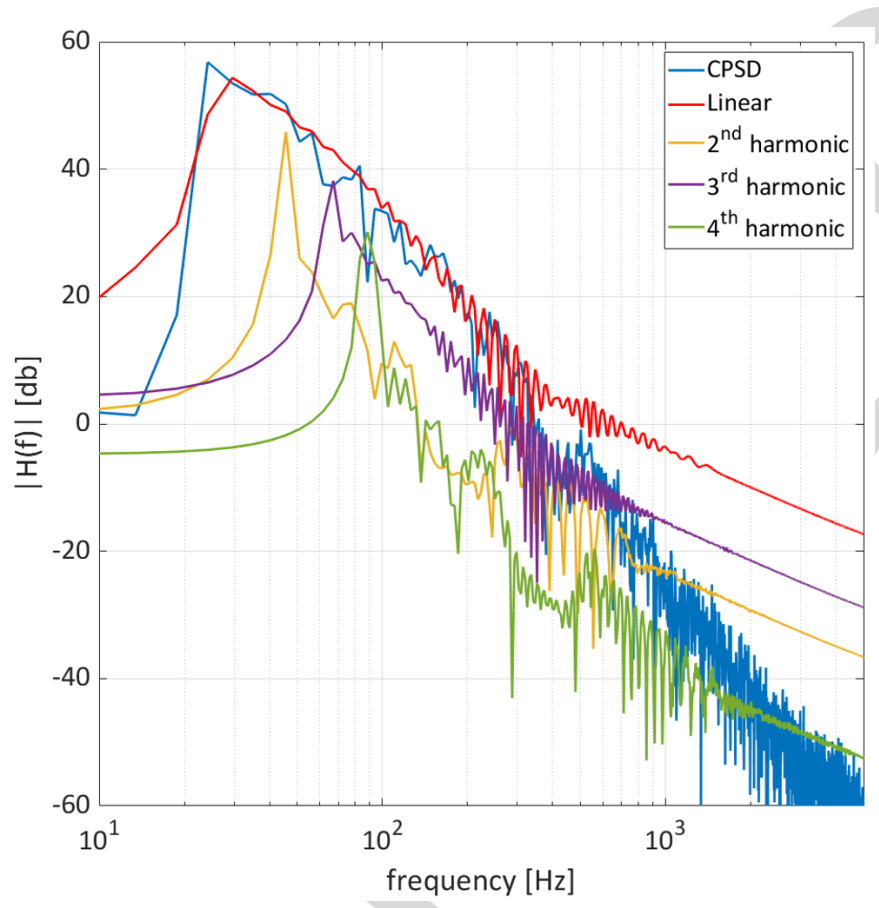

Fig. 27. CPSD, linear frequency response and harmonic frequency responses of the DUT, with measured T\&S parameters of table I.

are analysed too. For this reason, windows are applied to harmonic responses as well. As it can be noted from the Figure 27, the harmonic products affect the behaviour of the speaker mainly at low frequency, where the device is more susceptible to the non linear effects, in agreement with loudspeaker model theory.

\section{CONCLUSION}

In this paper a novel approach based on radar micro Doppler has been proposed for condition monitoring of loudspeakers in hostile environments. With the assumption of rigid body motion at low frequency, the displacement of a loudspeaker has been modelled as function of the frequency of the stimulus by considering the electro-mechanical components responsible of the dynamic response of the transducer, in both single tone and sine sweep analysis. In the first case, the phase, the spectrum and the magnitude square of the spectrogram of the received radar signal were compared to those from the model confirming that loudspeaker behaviour can be detected from radar. In particular, taking in account both micro-Doppler shift and the phase component of the received signal, the information of the displacement motion can be extracted. By increasing the voltage applied to the terminals of the driver, a resulting discrepancy between real and simulated signal appeared due to the non linear effects of the speaker. When the sine sweep test signal was used, some discrepancy between real and simulated signal appeared, as the rocking modes effect have not been taken in consideration in the displacement model. Nevertheless, the spectral analysis results demonstrates good ability in detecting irregular defects affecting the motion of the voice coil. Finally, a matched filter based approach was proposed to mechanically characterise the speaker. Cross power spectrum density, linear frequency response and harmonic frequency responses were analysed. These powerful indicator of possible manufacturing problems can be used as features for an automatic anomalies detection of loudspeaker defects. The proposed approach could be used in additional manufacturing applications. For example, in the same loudspeaker testing domain it could be integrated in Linear Suspension Testing aimed at assessing the quality control of moving parts. In other domains, the proposed technique could find application in testing of lightweight components (i.e.: made of carbon fiber) for aerospace use as well as in vibration analysis of machines such as the gearbox of a wind turbine.

\section{REFERENCES}

[1] W. Klippel, "Scanning vibrometer C5 hardware and software module of the klippel R\&D system," Klippel GmbH, Dresden, Germany, Tech. Rep., 2008. [Online]. Available: http://waf-e.dubudisk.com/ sangjinmedia.co.kr/sangjinmediacokr/O185KPZ/DubuDisk/www/R\&D\% 20System-09-01-C5_SCN_Scanning_Vibrometer.pdf

[2] W. Klippel and J. Schlechter, "Measurement and visualization of loudspeaker cone vibration," in Proc. Audio Eng. Soc. Conv, Oct. 2006, pp. 263-280. [Online]. Available: http://www.aes.org/e-lib/ browse.cfm?elib $=13716$

[3] W. Klippel, "Tutorial: Loudspeaker nonlinearities-Causes, parameters, symptoms," J. Audio Eng. Soc., vol. 54, no. 10, pp. 907-939, Oct. 2006 [Online]. Available: http://www.aes.org/e-lib/browse.cfm?elib=13881

[4] V. C. Chen, The Micro-Doppler Effect Radar. Norwood, MA, USA: Artech House, 2011. 
[5] C. Clemente, A. Balleri, K. Woodbridge, and J. J. Soraghan, "Developments in target micro-Doppler signatures analysis: Radar imaging, ultrasound and through-the-wall radar," EURASIP J. Adv. Signal Process. vol. 2013, no. 1, p. 47, Mar. 2013. doi: 10.1186/1687-6180-2013-47.

[6] D. Gaglione, C. Clemente, F. K. Coutts, G. Li, and J. J. Soraghan, "Model-based sparse recovery method for automatic classification of helicopters," in Proc. IEEE Radar Conf. (RadarCon), May 2015, pp. 1161-1165.

[7] C. Clemente, L. Pallotta, A. De Maio, J. J. Soraghan, and A. Farina, "A novel algorithm for radar classification based on Doppler characteristics exploiting orthogonal Pseudo-Zernike polynomials," IEEE Trans. Aerosp. Electron. Syst., vol. 51, no. 1, pp. 417-430, Jan. 2015.

[8] R. I. A. Harmanny, J. J. M. de Wit, and G. P. Cabic, "Radar microDoppler feature extraction using the spectrogram and the cepstrogram," in Proc. Eur. Radar Conf., Rome, Italy, Oct. 2014, pp. 165-168.

[9] A. R. Persico et al., "On model, algorithms, and experiment for microDoppler-based recognition of ballistic targets," IEEE Trans. Aerosp. Electron. Syst., vol. 53, no. 3, pp. 1088-1108, Jun. 2017.

[10] Y. Kim, S. Choudhury, and H.-J. Kong, "Application of micro-Doppler signatures for estimation of total energy expenditure in humans for walking/running activities,' IEEE Access, vol. 4, pp. 1560-1569, 2016.

[11] A. Assmann, A. Izzo, and C. Clemente, "Efficient micro-Doppler based pedestrian activity classification for ADAS systems using Krawtchouk moments," in Proc. 11th Int. Conf. Math. Signal Process. (IMA), Dec. 2016, pp. 1-6. [Online]. Available: https://strathprints.strath.ac.uk/ id/eprint/66617

[12] A. Izzo, L. Ausiello, C. Clemente, and J. J. Soraghan, "Radar microDoppler for loudspeaker analysis: An industrial process application," in Proc. Int. Conf. Radar Syst., Oct. 2017, pp. 1-6.

[13] L. L. Beranek and T. J. Mellow, "Electrodynamic loudspeakers," in Acoustics: Sound Fields and Transducers, L. L. Beranek and T. J. Mellow, Eds. New York, NY, USA: Academic, 2012, pp. 241-288. [Online]. Available: http://www.sciencedirect.com/science/article/pii/ B9780123914217000063

[14] G. Ni and S. J. Elliott, "Wave interpretation of numerical results for the vibration in thin conical shells," J. Sound Vibrat., vol. 333, no. 10, pp. 2750-2758, May 2014. [Online]. Available: https://eprints. soton.ac.uk/364781/

[15] W. Klippel and J. Schlechter, "Distributed mechanical parameters describing vibration and sound radiation of loudspeaker drive units," in Proc. Audio Eng. Soc. Conv., Oct. 2008, pp. 561-580. [Online]. Available: http://www.aes.org/e-lib/browse.cfm?elib=14683

[16] A. Farina, "Simultaneous measurement of impulse response and distortion with a swept-sine technique," in Proc. Audio Eng. Soc. Conv, Feb. 2000, pp. 1-24. [Online]. Available: http://www.aes.org/e-lib/ browse.cfm?elib=10211

[17] A. Farina, "Advancements in impulse response measurements by sine sweeps," in Proc. Audio Eng. Soc. Conv., May 2007, pp. 1626-1646. [Online]. Available: http://www.aes.org/e-lib/browse.cfm?elib=14106

[18] M. C. Bellini, L. Collini, A. Farina, D. Pinardi, and K. Riabova, "Measurement of loudspeakers with a laser Doppler vibrometer and the exponential sine sweep excitation technique," J. Audio Eng. Soc., vol. 65, nos. 7-8, pp. 600-612, Aug. 2017. [Online]. Available: http://www.aes.org/e-lib/browse.cfm?elib=19175

[19] M. A. Richards, J. A. Scheer, and W. A. Holm, Principles of Modern Radar, vol. 1. Rijeka, Croatia: SciTech, 2010. [Online]. Available: https://books.google.it/books?id=nD7tGAAACAAJ

[20] Audiomatica Srl. (2001). Clio Poket 2.0. [Online]. Available: http://www.audiomatica.com/wp/?page_id=2429

[21] B\&c Speakers. (1946). B \& C 10CL51 LF Driver. [Online]. Available: https://www.bcspeakers.com/en/products/lf-driver/10-0/8/10c151-8

Alessio Izzo received the B.Sc. (Laurea cum laude) and M.Sc. (Laurea Specialistica cum laude) degrees in telecommunication engineering and electronic engineering for automation and telecommunications from the Universita' degli Studi del Sannio, Benevento, Italy, in 2012 and 2015, respectively. During his master's degree, he was a Visiting Student at the Centre for Signal and Image Processing (CeSIP) Group, University of Strathclyde, Glasgow, U.K., where he is currently pursuing the Ph.D. degree working on adaptive filtering, audio signal processing, radar signal processing, micro-Doppler signature analysis, and classification based on deep learning techniques.
Ludovico Ausiello received the integrated master's degree in electronic engineering from the Universita' di Bologna, Bologna, Italy, and the Ph.D. degree focused on analog-to-digital converters, lossless data compression, and innovative high quality music formats including and surpassing the Super Audio CD standard from the Advanced Research Centre on Electronic System (ARCES) Group, Universita' di Bologna, in 2009. In 2009, he began a professional collaboration with Prof. A. Farina (Applied Acoustics at the University of Parma). He moved on to work for Harman Automotive as a System Engineer, then worked at Tannoy Ltd., as a Blue-Sky Researcher and a Transducer Designer, and then PSS Belgium as a Transducer Designer, while also starting his own consultancy firm, HouseYellow Acoustics and Musical Studies. In 2018, he became a Senior Lecturer in acoustics and audio engineering at Solent University, where he undertakes teaching and research. In 2019, he became a Fellow of the Higher Education Academy.

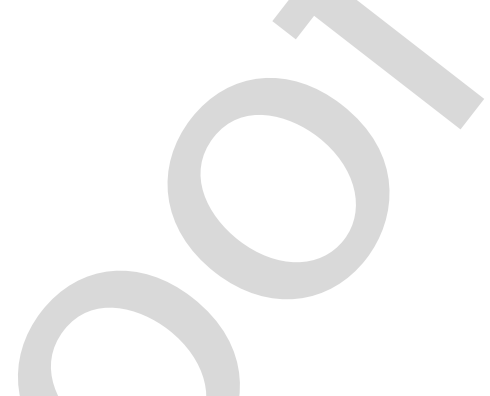

Carmine Clemente (S'09-M'13-SM'18) received the B.Sc. (Laurea cum laude) and M.Sc. (Laurea Specialistica cum laude) degrees in telecommunications engineering from the Universita degli Studi del Sannio, Benevento, Italy, in 2006 and 2009, respectively, and the Ph.D. degree from the Department of Electronic and Electrical Engineering, University of Strathclyde, Glasgow, U.K., in 2012. He is currently a Senior Lecturer with the Department of Electronic and Electrical Engineering, University of Strathclyde, where he is involved in advanced radar signal processing algorithm, MIMO radar systems, and micro-Doppler analysis. His research interests include synthetic aperture radar (SAR) focusing and bistatic SAR focusing algorithms development, micro-Doppler signature analysis and extraction from multistatic radar platforms, micro-Doppler classification, and statistical signal processing.

John J. Soraghan (S'83-M'84-SM'96) received the B.Eng. (Hons.) and M.Eng.Sc. degrees in electronic engineering from University College Dublin, Dublin, Ireland, in 1978 and 1983, respectively, and the Ph.D. degree in electronic engineering from the University of Southampton, Southampton, U.K., in 1989. After graduating, he worked at the Electricity Supply Board in Ireland and at Westinghouse Electric Corporation in the U.S. In 1986, he joined the Department of Electronic and Electrical Engineering, University of Strathclyde, Glasgow, U.K., as a Lecturer. He was the Manager of the Scottish Transputer Centre from 1988 to 1991, a Manager with the DTI Parallel Signal Processing Centre from 1991 to 1995 , and the Head of the ICSP from 2005 to 2007 . He became a Professor in signal processing in 2003 and held the Texas Instruments Chair in signal processing from 2004 to 2016 . He is currently the Director of the Sensor Signal Processing Research Groups with the Centre for Signal and Image Processing (CeSIP), University of Strathclyde. His doctoral research focused on synthetic aperture radar processing on the distributed array processor. 